\title{
Probing the initial conditions of high mass star formation
}

\section{Deuteration and depletion in high mass pre/protocluster clumps ${ }^{\star}$}

\author{
T. Pillai ${ }^{1, \star \star}$, F. Wyrowski ${ }^{1}$, J. Hatchell ${ }^{2}$, A. G. Gibb ${ }^{3}$, and M. A. Thompson ${ }^{4}$ \\ 1 Max-Planck-Institut für Radioastronomie, Auf dem Hügel 69, 53121 Bonn, Germany \\ e-mail: tpillai@cfa.harvard.edu \\ 2 School of Physics, University of Exeter, Stocker Road, Exeter EX4 4QL, UK \\ 3 Department of Physics \& Astronomy, University of British Columbia, Vancouver, BC, V6T 1Z1, Canada \\ ${ }^{4}$ Centre for Astrophysics Research, Science \& Technology Research Institute, University of Hertfordshire, College Lane, Hatfield, \\ AL10 9AB, UK
}

Received 23 May 2006 / Accepted 5 February 2007

\begin{abstract}
Aims. UltraCompact HII regions are signposts of high-mass star formation. Since high-mass star formation occurs in clusters, one expects to find even earlier phases of massive star formation in the vicinity of UltraCompact HII regions. Here, we study the amount of deuteration and depletion toward pre/protocluster clumps found in a wide-field $(10 \times 10 \mathrm{arcmin})$ census of clouds in 32 massive star-forming regions that are known to harbour UCHII regions.

Methods. We determine the column density of $\mathrm{NH}_{3}, \mathrm{NH}_{2} \mathrm{D}, \mathrm{CO}, \mathrm{H}^{13} \mathrm{CN}$, and $\mathrm{HC}^{15} \mathrm{~N}$ lines. We used the $(J, K)=(1,1)$ and $(2,2)$ inversion transitions of $\mathrm{NH}_{3}$ to constrain the gas temperatures.

Results. We find that $65 \%$ of the observed sources have strong $\mathrm{NH}_{2} \mathrm{D}$ emission and more than $50 \%$ of the sources exhibit a high degree of deuteration, $\left(0.1 \leq \mathrm{NH}_{2} \mathrm{D} / \mathrm{NH}_{3} \leq 0.7\right)$, 0.7 being the highest observed deuteration of $\mathrm{NH}_{3}$ reported to date. Our search for $\mathrm{NHD}_{2}$ in two sources did not result in a detection. The enhancement in deuteration coincides with moderate CO depletion onto dust grains. There is no evidence of a correlation between the two processes, though an underlying correlation cannot be ruled out as the depletion factor is very likely to be only a lower limit. Based on simultaneously observed $\mathrm{H}^{13} \mathrm{CN}$ and $\mathrm{HC}^{15} \mathrm{~N}(J=1-0)$ lines, we derive a high abundance ratio of $\mathrm{H}^{13} \mathrm{CN}$ to $\mathrm{HC}^{15} \mathrm{~N}$, which might indicate anomalous ratios of $\mathrm{C}$ and $\mathrm{N}$ isotopes relative to those derived toward the local ISM.

Conclusions. We find CO depletion and high deuteration towards cold cores in massive star forming regions. Therefore, these are good candidates for sources at the early phases of massive star formation. While our sensitive upper limits on $\mathrm{NHD}_{2}$ do not prove the predictions of the gas-phase and grain chemistry models wrong, an enhancement of $\approx 10^{4}$ over the cosmic $\mathrm{D} / \mathrm{H}$ ratio from $\mathrm{NH} \mathrm{H}_{2} \mathrm{D}$ warrants explanation.
\end{abstract}

Key words. stars: formation - astrobiology - molecular data - methods: observational - ISM: clouds - radio lines: ISM

\section{Introduction}

The earliest phases of high mass star formation are still poorly understood. The prevalent theory is that massive stars are born in dense clumps within giant molecular clouds (GMCs), where the presence of UltraCompact HII regions (UCHII) identifies massive stars which have already begun to ionize their surroundings. Observations (Cesaroni et al. 1994, 1998) have shown that these are often associated with warm $(T>100 \mathrm{~K})$, compact $(<0.1 \mathrm{pc})$ and very dense $\left(n_{\mathrm{H}_{2}}>10^{7} \mathrm{~cm}^{-3}\right)$ cores known as hot molecular cores (HMCs). An evolutionary sequence has been suggested based on recent observations (Codella et al. 2004; Beuther \& Shepherd 2005) with the HMC stage immediately preceding the formation of UCHII regions. But the stage before hot cores the precluster and early protocluster phases of massive star formation - have not yet been studied. Since many UCHII regions are located in clusters, one expects to find even earlier phases of massive star formation, and the raw material out of which

\footnotetext{
* Appendix $\mathrm{A}$ is only available in electronic form at http: //www . aanda. org

$\star \star$ Present address: Harvard-Smithsonian Centre for Astrophysics.
}

massive stars or star clusters form, in the vicinity of UCHIIs (Thompson et al. 2006).

This has prompted us to embark upon a program to search for massive pre/protocluster clumps by taking a wide-field $(10 \times$ 10 arcmin) census of clouds in 32 massive star-forming regions, harbouring UCHII regions (Wood \& Churchwell 1989). Our program is known as SCAMPS (the SCUBA Massive

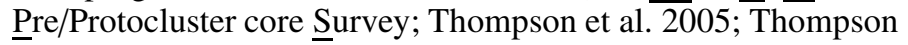
et al. 2007, in prep.).

We discovered a multitude of secondary, cold condensations and warmer clumps that might contain heavily embedded massive protostars. Many of the sources are seen as dark patches in MSX infrared images of the region, infrared dark clouds (IRDCs; Menten et al. 2005). As a result these clumps must have dust temperatures below $30 \mathrm{~K}$ (as evidenced by MIR upper limits) and have masses of a few 100 to $1000 M_{\odot}$. These clumps thus could be in a colder pre-protostellar phase.

In order to study the physical and chemical conditions of these clumps, we carried out a multi-wavelength survey toward them in various molecular tracers. In this study, we also included 9 sources which were selected on the basis of the MIR extinction (IRDCs) and followed up later with submillimeter dust 
continuum emission and millimeter rotational lines of $\mathrm{H}_{2} \mathrm{CO}$ (Carey et al. 1998). We have recently reported a study of ammonia in this sample (Pillai et al. 2006). Here, we report on our study using ammonia to probe the temperature and deuteration in the clumps and $\mathrm{CO}$ to estimate depletion of neutral molecules from the gas phase. Depletion has proved to be a good tracer of low-mass pre-stellar cores (Bacmann et al. 2003 and Crapsi et al. 2005), and $\mathrm{NH}_{3}$ is an important tool in measuring the physical conditions in molecular clouds and can be used to confirm the low temperatures and high densities required for depletion (Ho \& Townes 1983). Since only the lowest $\mathrm{NH}_{3}$ energy levels are expected to be populated for cold dark clouds $(T<20 \mathrm{~K})$, their physical conditions can be probed using the $(1,1)$ and $(2,2)$ inversion transitions in the metastable $(J, K)$ rotational levels of ammonia. Radiative transitions between different $K$-ladders are forbidden, therefore the lowest levels are populated only via collisions. The optical depth can be determined from the ratio of the hyperfine satellites. Thus, the population of the different levels can be estimated and hence the temperature of the gas determined.

$\mathrm{NH}_{3}$ is observed to be an excellent tracer of the dense gas where many other molecules would have heavily depleted (Tafalla et al. 2004). In addition, recent chemical models reveal that $\mathrm{NH}_{3}$ (and also $\mathrm{N}_{2} \mathrm{H}^{+}$), does not deplete from the gas phase for the densities observed in dark clouds $\left(<10^{6} \mathrm{~cm}^{-3}\right.$; Bergin \& Langer 1997). However in the light of recent experiments of the desorption of $\mathrm{CO}$ and $\mathrm{N}_{2}$ at temperatures relevant to dense cores, the relative difference between the derived $\mathrm{CO}$ and $\mathrm{N}_{2}$ binding energies is found to be significantly less than that currently adopted in astrochemical models (Öberg et al. 2005). Therefore, the observed non-depletion of $\mathrm{NH}_{3}$ and $\mathrm{N}_{2} \mathrm{H}^{+}$during the early formation of a massive cluster remains a "mystery".

High densities in the earliest phase are expected to enhance depletion of molecules (mainly CO) onto grains (Bacmann et al. 2003). The freeze out of abundant gas phase molecules along with the low temperatures $(\leq 20 \mathrm{~K})$ prevalent in these clumps leads to a high degree of deuteration (Flower et al. 2005; Roueff et al. 2005; Hatchell 2003; Shah \& Wootten 2001; Saito et al. 2000). Hence, the estimation of deuteration and depletion in clumps can be used as a chemical and physical chronometer.

In this paper we investigate the $\mathrm{NH}_{3}$ temperature and deuteration in our candidate pre/protocluster cores using observations with the Effelsberg $100 \mathrm{~m}$ and IRAM $30 \mathrm{~m}$ telescopes. In Sect. 2, we describe our single dish observations. We also discuss the data reduction and present the derived physical parameters for different molecules. In Sect. 3, we show that candidate pre/protocluster clumps exhibit very high deuteration and depletion. The fractionation of $\mathrm{NH}_{3}$ is compared with depletion of $\mathrm{CO}$ from the gas phase. The possible origin of deuterated species and the variation of deuteration with gas temperature is also discussed. HCN was also observed as part of the $30 \mathrm{~m}$ observations and we conclude the discussion with our interpretation of the $\mathrm{HCN}$ isotopic abundance with galactic distance.

\section{Observations and results}

In this section we present the results of single dish observations of $\mathrm{NH}_{3}, \mathrm{NH}_{2} \mathrm{D}, \mathrm{CO}$ and $\mathrm{HCN}$ lines on a sample of 23 SCAMPS sources and 9 IRDCs. The brightest compact submm sources with no MIR counterpart were selected for these observations from our original catalogue of the SCUBA fields. The parameters of the molecular transitions covered, along with the observing setups are given in Table 1. In Table 2, we give the source
Table 1. Parameters of observed rotational transitions.

\begin{tabular}{lrcc}
\hline \hline Species & Transition & $E_{1}^{a}(\mathrm{~K})$ & $v(\mathrm{MHz})$ \\
\hline $\mathrm{NH}_{3}$ & $(J, K=1,1)$ & 22.70 & 23694.496 \\
$\mathrm{NH}_{3}(J, K=2,2)$ & 63.89 & 23722.633 \\
$\mathrm{NH}_{2} \mathrm{D}$ (para) & $1_{11}-1_{01}$ & 16.55 & 85926.3 \\
$\mathrm{NH}_{2} \mathrm{D}$ (ortho) & $1_{11}-1_{01}$ & 15.98 & 110153.6 \\
$\mathrm{NHD}_{2}$ (ortho) & $1_{10}-1_{01}$ & 13.33 & 110812.9 \\
$\mathrm{NHD}_{2}$ (para) & $1_{10}-1_{01}$ & 13.09 & 110896.7 \\
$\mathrm{C}^{18} \mathrm{O}$ & $J=1-0$ & 0.0 & 109782.1734 \\
$\mathrm{C}^{18} \mathrm{O}$ & $J=2-1$ & 5.27 & 219560.3568 \\
$\mathrm{C}^{17} \mathrm{O}$ & $J=2-1$ & 5.39 & 224714.3850 \\
$\mathrm{H}^{13} \mathrm{CN}$ & $J=1-0, F=2-1$ & 0.0 & 86340.184 \\
$\mathrm{HC}$ & $J=1-0$ & 0.0 & 86054.961 \\
\hline
\end{tabular}

Note: ${ }^{a} E_{\mathrm{l}}$ is the lower energy level of the transition.

Table 2. Positions and velocities of the observed sources. IRDC positions are taken from Carey et al. (1998).

\begin{tabular}{|c|c|c|c|}
\hline Source & RA(2000) & $\operatorname{Dec}(2000)$ & $v_{\mathrm{LSR}}\left[\mathrm{km} \mathrm{s}^{-1}\right]$ \\
\hline \multicolumn{4}{|c|}{ SCAMPS } \\
\hline G8.13+0.25 & 18:02:55.69 & $-21: 47: 46.7$ & 19.4 \\
\hline G8.68-0.37 & 18:06:23.24 & $-21: 37: 14.1$ & $35.2 / 38.1$ \\
\hline G8.71-0.37 & 18:06:26.51 & $-21: 35: 46.6$ & 38.1 \\
\hline G10.21-0.31 & 18:09:20.63 & $-20: 15: 04.5$ & 12.8 \\
\hline G10.21-0.32 & 18:09:24.52 & $-20: 15: 41.4$ & 12.8 \\
\hline G10.15-0.34 & 18:09:21.38 & $-20: 19: 32.8$ & 12.8 \\
\hline G10.61-0.33 & 18:10:15.62 & $-19: 54: 46.6$ & 74.0 \\
\hline G12.19-0.12 & 18:12:41.67 & $-18: 25: 19.8$ & 27.6 \\
\hline G13.18+0.06 & 18:14:00.92 & $-17: 28: 41.2$ & 51.6 \\
\hline G15.01-0.67 & $18: 20: 21.22$ & $-16: 12: 42.2$ & 26.2 \\
\hline G15.03-0.65 & 18:20:18.80 & $-16: 11: 22.6$ & 26.2 \\
\hline G15.01-0.69 & $18: 20: 24.22$ & $-16: 13: 22.8$ & 26.2 \\
\hline G18.17-0.30 & $18: 25: 07.53$ & $-13: 14: 32.7$ & 54.9 \\
\hline G18.21-0.34 & $18: 25: 21.55$ & $-13: 13: 39.5$ & 54.9 \\
\hline G23.41-0.23 & $18: 34: 45.74$ & $-08: 34: 21.2$ & 104.2 \\
\hline G23.42-0.23 & 18:34:48.16 & $-08: 33: 56.1$ & 104.2 \\
\hline G23.44-0.18 & $18: 34: 39.25$ & $-08: 31: 36.2$ & 104.2 \\
\hline $\mathrm{G} 27.29+0.15$ & $18: 40: 34.70$ & $-04: 57: 18.1$ & 26.0 \\
\hline $\mathrm{G} 27.31+0.18$ & 18:40:32.45 & $-04: 55: 03.8$ & 26.0 \\
\hline G29.97-0.05 & $18: 46: 12.25$ & $-02: 39: 05.9$ & 100 \\
\hline G34.81-0.28 & $18: 53: 20.148$ & $+01: 28: 31.5$ & 59.0 \\
\hline G35.19-1.73 & 19:01:45.45 & $+01: 13: 21.5$ & 42.4 \\
\hline G81.74+0.59 & 20:39:00.37 & $+42: 24: 36.6$ & -3.1 \\
\hline \multicolumn{4}{|c|}{ IRDCs } \\
\hline G11.11-0.12 $\mathrm{NH}_{3} \mathrm{P} 1$ & $18: 10: 34.04$ & $-19: 21: 49.0$ & 29.2 \\
\hline G11.11-0.12 $\mathrm{NH}_{3} \mathrm{P} 3$ & 18:10:07.25 & $-19: 27: 28.0$ & 29.2 \\
\hline G11.11-0.12 $\mathrm{NH}_{3} \mathrm{P} 4$ & 18:10:07.25 & $-19: 28: 49.0$ & 29.2 \\
\hline $\mathrm{G} 19.30+0.07 \mathrm{NH}_{3} \mathrm{P} 1$ & 18:25:58.14 & $-12: 04: 4.9$ & 26.3 \\
\hline $\mathrm{G} 19.30+0.07 \mathrm{NH}_{3} \mathrm{P} 2$ & 18:25:52.69 & $-12: 04: 44.9$ & 26.3 \\
\hline $\mathrm{G} 28.34+0.06 \mathrm{NH}_{3} \mathrm{P} 1$ & $18: 42: 50.9$ & $-04: 03: 13.9$ & 78.4 \\
\hline $\mathrm{G} 28.34+0.06 \mathrm{NH}_{3} \mathrm{P} 2$ & $18: 42: 52.4$ & $-03: 59: 53.9$ & 78.4 \\
\hline G33.71-0.01 & 18:52:53.81 & $+00: 41: 06.4$ & 104.2 \\
\hline G79.34+0.33 & $20: 32: 26.20$ & $+40: 19: 40.9$ & 0.1 \\
\hline
\end{tabular}

list. The derived line parameters and the column densities are presented in Tables 3-6.

\subsection{IRAM $30 \mathrm{~m}$ observations}

The $\mathrm{NH}_{2} \mathrm{D}, \mathrm{C}^{18} \mathrm{O}, \mathrm{H}^{13} \mathrm{CN}$ and $\mathrm{HC}^{15} \mathrm{~N}$ observations were made at the IRAM $30 \mathrm{~m}$ telescope $^{1}$ on Pico Veleta in August 2003

\footnotetext{
${ }^{1}$ IRAM is an international institute for research in millimeter astronomy. IRAM is supported by INSU/CNRS (France), MPG (Germany) and IGN (Spain).
} 
toward the 32 candidate pre/protocluster clumps given in Table 2 . We performed 9 point maps with $10^{\prime \prime}$ spacing around the peak to be able to compare, independently of the beam, lines at different frequencies. All line parameters listed are averaged over the map.

Individual positions were observed with an integration time of 2 minutes per position in the position switch mode. The receivers $\mathrm{A} 100, \mathrm{~B} 100, \mathrm{~A} 230$ and $\mathrm{B} 230$ were tuned to 86.13, 109.975, 219.56 and $231.32 \mathrm{GHz}$ respectively.

The VESPA autocorrelator was used at the backend, with a spectral resolution of $40 \mathrm{kHz}$ and $320 \mathrm{kHz}$ at 100 and $230 \mathrm{GHz}$ respectively. We had average summer weather conditions with a maximum system temperature of $248 \mathrm{~K}$ at $110 \mathrm{GHz}$. Towards selected sources we also have $\mathrm{C}^{17} \mathrm{O}(J=2-1)$ and $\mathrm{NHD}_{2}\left(1_{10^{-}}\right.$ $\left.1_{01}\right)$ observations. The half power beam width $(H P B W)$ of the $30 \mathrm{~m}$ telescope is $22^{\prime \prime}$ at $110 \mathrm{GHz}$ and $11^{\prime \prime}$ at $230 \mathrm{GHz}$. The main beam efficiency at these frequencies is 0.75 and 0.52 , respectively.

\subsection{Effelsberg $100 \mathrm{~m}$ observations}

We observed the $\mathrm{NH}_{3}(1,1)$ and $(2,2)$ transitions with the Effelsberg $100 \mathrm{~m}$ telescope ${ }^{2}$ in April 2004 for the 23 sources listed in Table 2. Two of the sources were re-observed because of baseline problems with the receiver. With the AK 8192 backend, we were able to observe the $(1,1),(2,2),(3,3)$ and $(4,4)$ transitions in both polarisations simultaneously using the $K$-band receiver. With 8 subunits of $10 \mathrm{MHz}$ bandwidth, the resulting spectral resolution was $\approx 0.25 \mathrm{~km} \mathrm{~s}^{-1}$. The beam at the $\mathrm{NH}_{3}$ frequencies was $40^{\prime \prime}$. The observations were performed in the frequency switching mode. Pointing was checked at hourly intervals by continuum scans on G10.62. We estimate the pointing to be accurate to within 6 ". The pointing scans were used for the absolute flux calibration. The $\mathrm{NH}_{3}$ observations toward the 9 IRDCs are reported in Pillai et al. (2006). Note that for G12.190.12 and G29.97-0.05 we report the observations carried out in October 2002 with a spectral resolution of $\approx 1 \mathrm{~km} \mathrm{~s}^{-1}$.

\subsection{Results of the $\mathrm{NH}_{3}$ and $\mathrm{NH}_{2} \mathrm{D}$ single dish observations}

The ratio of the brightness temperatures of the $\mathrm{NH}_{3}(1,1)$ and $(2,2)$ transitions, along with the optical depth, can be used to estimate the rotational temperature. For temperatures $<20 \mathrm{~K}$, which are typical for cold dark clouds, the rotational temperature closely follows the gas kinetic temperature (Walmsley \& Ungerechts 1983). Like $\mathrm{NH}_{3}$, its isotopologue $\mathrm{NH}_{2} \mathrm{D}$ also has hyperfines which allows the estimation of optical depth and hence the column density. Thus the fractionation ratio can be estimated assuming that $\mathrm{NH}_{2} \mathrm{D}$ and $\mathrm{NH}_{3}$ are co-spatial and hence have the same gas temperature.

Out of the 32 sources that were observed, $\mathrm{NH}_{2} \mathrm{D}$ with hyperfines were detected in 22 sources with $\mathrm{S} / \mathrm{N}$ ratio $>5 \sigma$ while we have a $100 \% \mathrm{NH}_{3}(1,1)$ and $(2,2)$ detection. While we detected the $(3,3)$ lines in most of the sources, the $(4,4)$ lines were not detected. The fits to the hyperfines for $\mathrm{NH}_{3}$ and $\mathrm{NH}_{2} \mathrm{D}$ were done using CLASS methods $\mathrm{NH}_{3}(1,1)$ and HFS respectively (Forveille et al. 1989). The line parameters from the resultant fits are given ${ }^{3}$ in Tables A.2 and A.3. For those sources with a

\footnotetext{
${ }^{2}$ Based on observations with the $100 \mathrm{~m}$ telescope of the MPIfR (Max-Planck-Institut für Radioastronomie) at Effelsberg).

3 Table A.2, available in electronic form, contains the following information. Column 1 lists the name of the source, Cols. 2 and 5 gives the $\mathrm{NH}_{3}(1,1)$ and $(2,2)$ main beam brightness temperature respectively,
}

high uncertainty in the $\mathrm{NH}_{2} \mathrm{D}$ optical depth, the main line is fitted with a single Gaussian and the integrated intensity is quoted. The spectra are shown in Fig. A.1.

The basic physical parameters, namely the rotational temperature, the kinetic temperature and ammonia column density were derived using the standard formulation for $\mathrm{NH}_{3}$ spectra (Bachiller et al. 1987). The expressions used to estimate the column densities from the radiative transfer equations for all other molecules including $\mathrm{NH}_{2} \mathrm{D}$ are given in Appendix A. The deduced physical parameters are given in Table 3 . The uncertainties given in brackets are formal errors obtained by a Gaussian error propagation. We have derived the $\mathrm{NH}_{2} \mathrm{D}$ column density solely based on the ortho transition. We assume that the ortho and para transitions are in LTE.

For the few cases with significant detection of the para state we find that ratio of the integrated line intensity $\left(\int T_{\mathrm{MB}} \mathrm{d} v\right)$ is approximately 3 consistent with a thermalised ortho to para ratio of 3 .

For the column density determination of $\mathrm{NH}_{2} \mathrm{D}$, we have averaged over the 9 points of the map to compare to the $\mathrm{NH}_{3}$ results. Essentially the resolution of the $\mathrm{NH}_{2} \mathrm{D}$ observations has been degraded to that of the $\mathrm{NH}_{3}$ observations. A caveat is that a map obtained with $10^{\prime \prime}$ spacing at the IRAM $30 \mathrm{~m}$ does not completely sample the $40^{\prime \prime}$ beam of the Effelsberg telescope. However, it is very unlikely that there is significant emission outside the beam of the Effelsberg telescope (i.e. its $F W H M$ ) because a)we find that the brightest peak is always at the center of the map, b) box averaging delivers a similar result to a $1 \mathrm{D}$ Gaussian weighted average c) the peak dust emission positions have been chosen as the centre coordinates for these observations.

The rotational temperatures are in a range from 11 to $23 \mathrm{~K}$, with ammonia column densities from 1 to $5 \times 10^{15} \mathrm{~cm}^{-3}$. We obtain $\left[\mathrm{NH}_{2} \mathrm{D} / \mathrm{NH}_{3}\right]$ ratios from $0.004-0.66$. A deuteration factor of $66 \%$ in $\mathrm{NH}_{3}$ is much larger than the largest reported value so far, $\sim 33 \%$ by Hatchell (2003). In 10 out of the 22 sources with $\mathrm{NH}_{2} \mathrm{D}$ detection, we obtain abundance ratios $\leq 0.02$ while the rest of the sources have a high degree of deuteration $(\geq 13 \%)$. These 10 sources with low ratios show optically thin $\mathrm{NH}_{2} \mathrm{D}$ emission.

In Fig. 1, the correlation between the velocities and line widths of the $\mathrm{NH}_{2} \mathrm{D}$ and $\mathrm{NH}_{3}$ emission is shown. Velocities and line widths are clearly different in some sources, with the $\mathrm{NH}_{2} \mathrm{D}$ line widths being smaller in most cases. This could be either due to $\mathrm{NH}_{2} \mathrm{D}$ and $\mathrm{NH}_{3}$ tracing different regions or the slightly different beams at the two frequencies, in which case, clumping might contribute to a larger line width. It is interesting to note that the critical density of the reported $\mathrm{NH}_{2} \mathrm{D}$ transition is a factor 47 higher than that of the corresponding $\mathrm{NH}_{3}$ transition, assuming similar collisional rates. However, a more likely explanation is that $\mathrm{NH}_{3}$ could be originating from the more disturbed protocluster environment, while $\mathrm{NH}_{2} \mathrm{D}$ might be tracing the gas in precluster cores (Pillai et al. in prep.).

\subsection{Results of the CO observations}

The $\mathrm{C}^{18} \mathrm{O}(1-0)$ and (2-1) observations were performed simultaneously with $\mathrm{NH}_{2} \mathrm{D}\left(1_{1,1}-1_{0,1}\right)$. Figure A.2 shows the spectra toward all sources. Note that there are secondary features in

Col. 3 gives the $(1,1)$ line width, and Col. 4 lists the $(1,1)$ main group optical depth. In Table A.3 we list the sources observed in $\mathrm{NH}_{2} \mathrm{D}$, the integrated line intensity, FWHM of the line and the total optical depth from Cols. 1 to 4 . 
Table 3. $\mathrm{NH}_{2} \mathrm{D}$ and $\mathrm{NH}_{3}$ column densities, rotational temperature and fractionation.

\begin{tabular}{lccll}
\hline \hline Source & $\begin{array}{c}T_{\text {rot }}{ }^{a} \\
\mathrm{~K}\end{array}$ & $\begin{array}{c}N_{\mathrm{NH}_{3}}{ }^{b} \\
10^{15}\left(10^{14}\right) \mathrm{cm}^{-2}\end{array}$ & $\begin{array}{l}N_{\mathrm{NH}_{2} \mathrm{D}}{ }^{c} \\
10^{13} \mathrm{~cm}^{-2}\end{array}$ & {$\left[\mathrm{NH}_{2} \mathrm{D} / \mathrm{NH}_{3}\right]^{d}$} \\
\hline G8.13+0.25 & $18.0(3.0)$ & $1.8(0.4)$ & $1.7(0.1)$ & $1.0(0.2)$ \\
G8.68-0.37 & $17.4(2.3)$ & $1.6(0.3)$ & $2.4(0.1)$ & $1.5(0.3)$ \\
G8.71-0.37 & $12.1(0.8)$ & $1.3(0.1)$ & $39.4(12.4)$ & $30.3(9.8)$ \\
G10.21-0.31 & $15.4(1.5)$ & $1.5(0.2)$ & $59.7(10.6)$ & $39.8(8.9)$ \\
G10.21-0.32 & $14.1(0.7)$ & $2.1(0.3)$ & $70.9(29.9)$ & $33.7(15.0)$ \\
G10.61-0.33 & $17.8(1.5)$ & $2.3(0.1)$ & $51.3(22.3)$ & $24.4(10.7)$ \\
G11.11-0.12P1 & $12.5(1.2)$ & $1.4(0.2)$ & $1.0(0.1)$ & $0.4(0.1)$ \\
G11.11-0.12P4 & $10.6(1.6)$ & $1.8(0.5)$ & $1.7(0.3)$ & $38.6(17.7)$ \\
G12.19-0.12 & $12.6(0.3)$ & $4.4(0.2)$ & $5.6(0.2)$ & $1.3(0.3)$ \\
G13.18+0.06 & $17.4(1.3)$ & $2.8(0.2)$ & $88.9(23.2)$ & $31.7(8.6)$ \\
G18.17-0.30 & $15.8(1.2)$ & $1.8(0.2)$ & $119.7(31.5)$ & $66.5(19.0)$ \\
G18.21-0.34 & $15.5(1.2)$ & $1.7(0.2)$ & $47.3(19.7)$ & $27.8(12.0)$ \\
G23.41-0.23 & $18.8(1.8)$ & $2.5(0.3)$ & $77.7(51.3)$ & $31.1(20.8)$ \\
G23.44-0.18 & $23.3(1.5)$ & $5.2(0.4)$ & $5.0(0.2)$ & $1.0(0.1)$ \\
G27.29+0.15 & $17.8(1.7)$ & $3.0(0.3)$ & $1.4(0.2)$ & $0.5(0.1)$ \\
G28.34+0.06P2 & $15.7(2.0)$ & $2.2(0.3)$ & $4.1(0.2)$ & $1.9(0.3)$ \\
G29.97-0.05 & $13.7(1.4)$ & $2.2(0.3)$ & $67.7(15.2)$ & $30.8(8.1)$ \\
G33.71-0.01 & $19.5(4.6)$ & $3.0(0.8)$ & $1.4(0.1)$ & $0.5(0.1)$ \\
G34.81-0.28 & $16.6(1.0)$ & $1.6(0.1)$ & $2.7(0.2)$ & $1.7(0.2)$ \\
G35.19-1.73 & $17.0(0.9)$ & $1.8(0.1)$ & $74.0(11.0)$ & $41.1(6.5)$ \\
G79.34+0.33 & $13.7(1.0)$ & $1.4(0.1)$ & $17.9(15.9)$ & $12.8(11.4)$ \\
G81.74+0.59 & $18.4(1.1)$ & $2.7(0.2)$ & $92.9(17.6)$ & $34.4(7.0)$ \\
\hline
\end{tabular}

${ }^{a}$ Rotational temperature derived from $\mathrm{NH}_{3}$ observations. ${ }^{b} \mathrm{NH}_{3}$ column density. ${ }^{c} \mathrm{NH}_{2} \mathrm{D}$ column density. ${ }^{d}$ Ratio of $\mathrm{NH}_{3}$ and $\mathrm{NH}_{2} \mathrm{D}$ column densities in percentage. Note: similar notations are used for other molecules elsewhere in this paper.
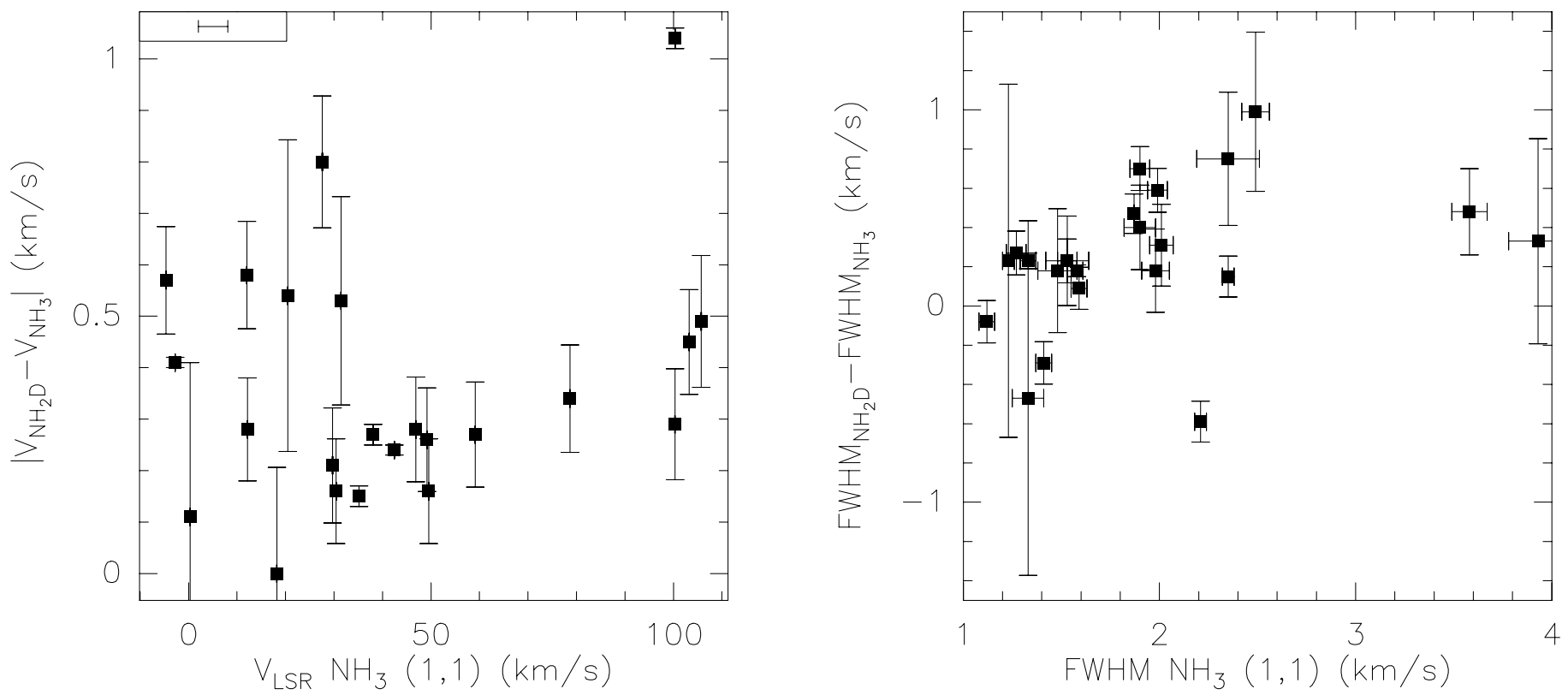

Fig. 1. Left panel: comparison of the LSR velocities of $\mathrm{NH}_{3}$ and $\mathrm{NH}_{2} \mathrm{D}$. The median spectral resolution after Hanning smoothing is marked in the upper left corner of the panel. Right panel: comparison of $\mathrm{NH}_{3}$ and $\mathrm{NH}_{2} \mathrm{D}$ line widths. Points with no y error bars are those for which the line width has been fixed based on the hfs fit to the optically thin emission, to derive the $\mathrm{NH}_{2} \mathrm{D}$ integrated intensity.

the $\mathrm{C}^{18} \mathrm{O}$ spectra for a few sources, likely to be line of sight components not seen in dense gas like $\mathrm{NH}_{3}$. In Table A. $4^{4}$, we list the observed line parameters based on Gaussian fits, and, in Table 4, the column density and excitation temperature estimates (see Appendix A) and the $\mathrm{C}^{18} \mathrm{O}$ abundances. The $\mathrm{C}^{18} \mathrm{O}$

4 Table A.4, available in electronic form, contains the following information. Column 1 lists the name of the source, Cols. 2 and 3 gives the $\mathrm{C}^{18} \mathrm{O}(1-0)$ and $(2-1)$ integrated intensity. excitation temperature has been derived from the $\mathrm{C}^{18} \mathrm{O} J=1-0$ and $J=2-1$ line ratios.

Based on the dust continuum and $\mathrm{CO}$ observations for the observed sample, the depletion can be studied for the first time on a sample of massive clumps. Recent observational studies on the condensation of $\mathrm{CO}$ in low mass prestellar cores reveal that in the initial cold and dense evolutionary stage, $\mathrm{CO}$ is heavily 

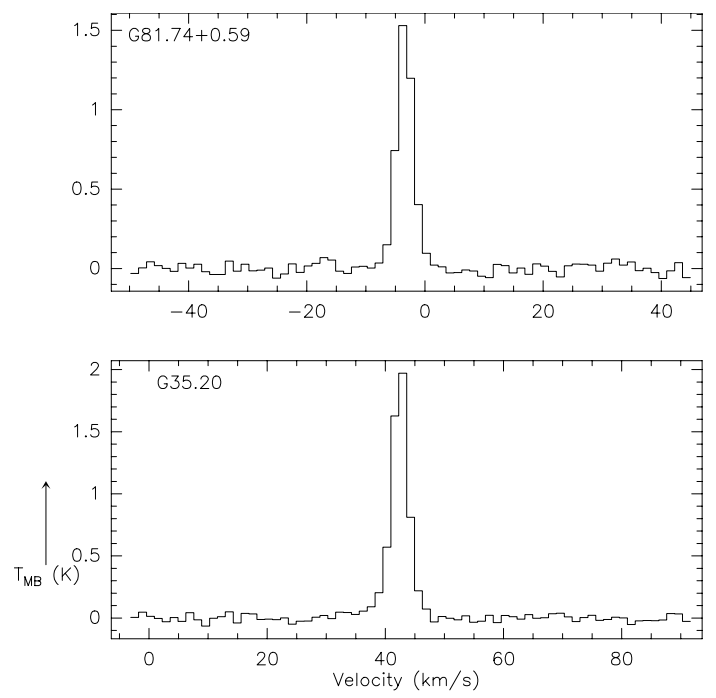

Fig. 2. $\mathrm{C}^{17} \mathrm{O}(J=2-1)$ spectra toward G35.19-1.73 and G81.74+0.59 observed with the IRAM $30 \mathrm{~m}$ telescope.

depleted onto the dust grains (Bacmann et al. 2002, Savva et al. 2003). The depletion factor $\eta$ is defined as

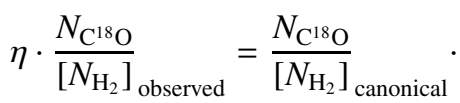

The $\mathrm{C}^{18} \mathrm{O}$ column density, $N_{\mathrm{C}^{18} \mathrm{O}}$, is derived from the observed integrated intensity (see Appendix A) and assumimg that the gas temperature is equal to the excitation temperature derived from the $\mathrm{C}^{18} \mathrm{O}$ line ratios.

The effective $\mathrm{H}_{2}$ column density (Launhardt 1996) is calculated from

$N\left(\mathrm{H}_{2}\right)=\frac{6.2 \times 10^{16} S_{v} \lambda^{3} \mathrm{e}^{\frac{1.44 \times 10^{4}}{\mathrm{~T}_{\mathrm{d}} \lambda}}}{\kappa_{\mathrm{m}}(\lambda) \theta^{2}} \frac{Z_{\odot}}{\mathrm{Z}}$

where $S_{v}$ is the flux density in Jy/beam. $\lambda$ the wavelength in $\mu \mathrm{m}$, $\mathrm{Z} / \mathrm{Z}_{\odot}$ is the metalicity relative to the solar metalicity (we assume 1) and $\theta$ is the $F W H M$ of the beam at wavelength $\lambda$. We used an opacity $\kappa_{\mathrm{m}}$ of $1.85 \mathrm{~cm}^{2} / \mathrm{g}$ of dust at $850 \mu \mathrm{m}$ for dust grains with thick ice mantles and gas density $n(\mathrm{H})=10^{6} \mathrm{~cm}^{-3}$ (Ossenkopf \& Henning 1994). The column density was derived after smoothing the $850 \mu \mathrm{m}$ dust continuum emission to the $20^{\prime \prime}$ resolution of the $\mathrm{C}^{18} \mathrm{O}$ data (Thompson et al. 2007, in prep).

In these dense clumps it is possible that the $\mathrm{C}^{18} \mathrm{O}$ line may be optically thick and hence derived column densities could be a lower limit. In order to investigate this possibility, we observed the rarer isotopomer $\mathrm{C}^{17} \mathrm{O}$ toward two of our sources, G35.191.73 and G81.74+0.59 as shown in Fig. 2. The line parameters are given in Table 5. In Sect. 3.1.1, we discuss how $\mathrm{C}^{17} \mathrm{O}$ is used as a more reliable tracer of the optical depth.

\subsection{Results of the $\mathrm{H}^{13} \mathrm{CN}$ and $\mathrm{HC}^{15} \mathrm{~N}$ observations}

We also observed the isotopomeric species of $\mathrm{HCN}, \mathrm{H}^{13} \mathrm{CN}$ and $\mathrm{HC}^{15} \mathrm{~N}$ in our $3 \mathrm{~mm}$ setup with the IRAM $30 \mathrm{~m}$ telescope. $\mathrm{H}^{13} \mathrm{CN}$ was detected in almost all sources except G19.30P1/P2 and G10.15-0.34. In Fig. A.3, we show the spectra toward all sources where both $\mathrm{H}^{13} \mathrm{CN}$ and $\mathrm{HC}^{15} \mathrm{~N}$ are detected with sufficient $\mathrm{S} / \mathrm{N}(\geq 3 \sigma)$. The $\mathrm{H}^{13} \mathrm{CN}$ line has hyperfines, which allows to estimate the optical depth. However, we find that $\mathrm{H}^{13} \mathrm{CN}$ is optically thin in almost all cases. In two sources with moderate optical depths, the uncertainty in $\tau$ is too large to derive meaningful constraints on the column density. The line parameters are given in Table 6 along with their column densities. The column density is computed using the gas temperature derived from $\mathrm{NH}_{3}$ as the excitation temperature (see Appendix A).

\section{Analysis and discussion}

\subsection{CO depletion}

The derived $\mathrm{C}^{18} \mathrm{O}$ abundances are a factor of 2-12 lower than the canonical value of $1.7 \times 10^{-7}$ (Frerking et al. 1982). Wu \& Yang (2005) find similar depletion factors for the infrared dark cloud G79.2+0.38, another of the clumps in the cloud in the present study.

\subsubsection{Is $\mathrm{C}^{18}$ optically thin?}

As mentioned in Sect. 2.4, the CO column density as determined from $\mathrm{C}^{18} \mathrm{O}$ transitions might be underestimated if $\mathrm{C}^{18} \mathrm{O}$ is optically thick. However, the rarer isotopomer $\mathrm{C}^{17} \mathrm{O}$ is expected to be optically thin. The canonical value of the relative abundance of $\mathrm{C}^{18} \mathrm{O}$ w.r.t $\mathrm{C}^{17} \mathrm{O}, A(18,17)$, is 3.65 (Wilson \& Rood 1994) in the ISM. As discussed in Kramer et al. (1999), the measured ratios of the integrated intensities of the $\mathrm{C}^{18} \mathrm{O}$ and $\mathrm{C}^{17} \mathrm{O}$ lines can then be used to determine the $\mathrm{C}^{18} \mathrm{O}$ optical depth.

Due to the limited observing time, we observed the $\mathrm{C}^{17} \mathrm{O}$ line only in the two brightest $\mathrm{C}^{18} \mathrm{O}$ sources. If $R^{18,17}$ and $\tau_{18}^{18,17}$ denotes the ratio of the integrated intensities of the $\mathrm{C}^{18} \mathrm{O}$ and $\mathrm{C}^{17} \mathrm{O}$ lines and the optical depth of $\mathrm{C}^{18} \mathrm{O}$ as derived from the ratio, then

$R^{18,17}=\frac{1-\exp \left(-\tau_{18}^{18,17}\right)}{1-\exp \left(-\tau_{18}^{18,17} / A(18,17)\right)}$.

The assumptions involved in deriving Eq. (3), are that both isotopomers have the same excitation temperatures and beam filling factors.

For G35.19-1.73, we find that $\tau_{18}^{18,17}=1.35$, which implies that the correction factor for the column density given by $\tau /(1-\exp (-\tau))=1.8$, is clearly smaller than the observed depletion. However, for G81.74+0.59, the ratio itself $R^{18,17} \sim 6$, suggesting that Eq. (3) is not valid anymore. It is unlikely to be due to different excitation conditions or the extent of emission of $\mathrm{C}^{18} \mathrm{O}$ and $\mathrm{C}^{17} \mathrm{O}$. It is indeed possible that there are real variations in the ratio of ${ }^{18} \mathrm{O}$ to ${ }^{17} \mathrm{O}$, a possibility which needs to be investigated. Additionally, the observed relatively small observed peak brightness temperatures for $\mathrm{C}^{18} \mathrm{O}$ exclude high optical depth in this line. Therefore, to the extent that we assume G35.19-1.73 to be representative of our sample, we may conclude that $\mathrm{C}^{18} \mathrm{O}$ optically depth is "moderate".

\section{2. $\mathrm{NH}_{3}$ deuteration}

How do we account for the high deuteration we observe in these pre/protostellar clumps? There are two main pathways to bring about deuteration (Rodgers \& Charnley 2001; Millar 2002, 2003; Roueff et al. 2005); a) gas-phase reactions, b) production of deuterium bearing molecules on grain surfaces. Accretion of neutrals onto the dust grains that would otherwise destroy $\mathrm{H}_{2} \mathrm{D}^{+}$ enhances deuteration. Up to now all these processes have been used to explain fractionation in different sources.

In pre/protostellar cores, ion-molecule exchange reactions prevalent at low temperatures coupled with the depletion of $\mathrm{CO}$ 
Table 4. $\mathrm{C}^{18} \mathrm{O}$ column density and abundance from $(J=1-0)$ and $(J=2-1)$ transitions.

\begin{tabular}{|c|c|c|c|c|c|}
\hline Source & $T_{\mathrm{ex}}$ & $N_{\mathrm{C}^{18} \mathrm{O}}$ & $N\left(\mathrm{H}_{2}\right)^{a}$ & $\chi_{\mathrm{C}^{18} \mathrm{O}}^{b}$ & $\eta^{c}$ \\
\hline & $\mathrm{K}$ & $10^{16}\left(10^{15}\right) \mathrm{cm}^{-2}$ & $10^{23} \mathrm{~cm}^{-2}$ & $10^{-8}$ & \\
\hline G8.13+0.25 & 11.8 & $1.6(0.1)$ & 2.6 & 6.2 & 2.7 \\
\hline G8.68-0.37 & 8.5 & $2.4(0.9)$ & 7.9 & 3.0 & 5.6 \\
\hline G8.71-0.37 & 4.2 & $1.8(0.5)$ & 3.5 & 5.2 & 3.2 \\
\hline G10.15-0.34 & 19.1 & $2.1(0.4)$ & 3.8 & 5.4 & 3.1 \\
\hline G10.21-0.31 & 8.4 & $1.4(0.2)$ & 2.2 & 6.4 & 2.7 \\
\hline G10.21-0.32 & 8.0 & $2.6(0.3)$ & 7.4 & 3.6 & 4.8 \\
\hline G10.61-0.33 & 9.1 & $1.0(0.3)$ & 2.7 & 3.8 & 4.5 \\
\hline G11.11-0.12P1 & 6.4 & $0.6(0.1)$ & 1.1 & 5.5 & 3.1 \\
\hline G11.11-0.12P3 & 6.9 & $0.5(0.3)$ & 0.8 & 6.2 & 2.7 \\
\hline G11.11-0.12P4 & 6.6 & $0.5(0.2)$ & 1.4 & 3.5 & 4.8 \\
\hline G12.19-0.12 & 11.6 & $1.2(0.2)$ & 2.4 & 5.1 & 3.3 \\
\hline G13.18+0.06 & 15.7 & $1.2(0.6)$ & 6.3 & 1.8 & 9.3 \\
\hline G15.01-0.67 & 32.5 & $6.0(0.4)$ & 16.6 & 3.6 & 4.7 \\
\hline G15.01-0.69 & 52.2 & $4.5(0.6)$ & 11.6 & 3.9 & 4.4 \\
\hline G15.03-0.65 & 51.7 & $5.5(0.6)$ & 14.2 & 3.9 & 4.4 \\
\hline G18.17-0.30 & 14.3 & $1.5(0.8)$ & 3.3 & 4.6 & 3.7 \\
\hline G18.21-0.34 & 10.9 & $0.7(0.4)$ & 2.5 & 3.0 & 5.8 \\
\hline G23.41-0.23 & 8.4 & $3.4(0.8)$ & 9.3 & 3.6 & 4.7 \\
\hline G23.42-0.23 & 9.0 & $3.1(0.3)$ & 3.8 & 8.0 & 2.1 \\
\hline G23.44-0.18 & 9.2 & $3.0(0.5)$ & 7.7 & 3.9 & 4.3 \\
\hline $\mathrm{G} 27.29+0.15$ & 8.8 & $1.2(0.2)$ & 1.7 & 6.9 & 2.5 \\
\hline $\mathrm{G} 27.31+0.18$ & 8.3 & $0.5(0.2)$ & 1.7 & 2.7 & 6.3 \\
\hline $\mathrm{G} 28.34+0.06 \mathrm{P} 1$ & 4.8 & $1.3(0.5)$ & 1.5 & 8.6 & 2.0 \\
\hline $\mathrm{G} 28.34+0.06 \mathrm{P} 2$ & 8.7 & $1.4(0.5)$ & 7.8 & 1.8 & 9.6 \\
\hline G29.97-0.05 & 9.3 & $1.3(0.7)$ & 2.6 & 4.8 & 3.5 \\
\hline G79.34+0.33 & 8.4 & $0.7(0.1)$ & 2.1 & 3.5 & 4.9 \\
\hline G81.74+0.59 & 16.0 & $1.8(0.2)$ & 4.7 & 3.8 & 4.4 \\
\hline
\end{tabular}

${ }^{a} \mathrm{H}_{2}$ column density derived from the $850 \mu \mathrm{m}$ dust continuum flux measured using SCUBA. The dust temperature is assumed to be equal to the gas temperature derived from $\mathrm{NH}_{3}(1,1)$ and $(2,2)$ measurements as given in Table $3 .{ }^{b}$ abundance of $\mathrm{C}^{18} \mathrm{O}$ relative to $\mathrm{H}_{2} .{ }^{c}$ depletion factor compared to the canonical value of $1.7 \cdot 10^{-7}$ (Frerking et al. 1982).

Table 5. $\mathrm{C}^{17} \mathrm{O}(J=2-1)$ line parameters.

\begin{tabular}{lcc}
\hline \hline Source & $\int_{\mathrm{K}} T_{\mathrm{MB}} \mathrm{d} v$ & $\begin{array}{c}\Delta v \\
\mathrm{Km} \mathrm{s}^{-1}\end{array}$ \\
\hline G35.19-1.73 & $7.1(0.1)$ & $3.3(0.1)$ \\
G81.74+0.59 & $5.5(0.1)$ & $3.3(0.1)$ \\
\hline
\end{tabular}

from gas phase is preferred over grain production of highly deuterated species at temperatures of $20 \mathrm{~K}$ (Shah \& Wootten 2001; Caselli et al. 2003). The freeze-out of molecules from the gas onto the dust grains, particularly that of heavy molecules like $\mathrm{CO}$ in the cold initial phase has been predicted by chemical models (Flower et al. 2005; Rodgers \& Charnley 2001; Roberts \& Millar 2000a,b; Brown \& Millar 1989b; Watson 1976). These predictions have been validated by observations of molecular freeze out onto dust grains in low-mass starless dense cores (Caselli et al. 1999; Tafalla et al. 2002). The primary fractionation reaction that dominates at low temperatures $(<20 \mathrm{~K})$ is

$\mathrm{H}_{3}^{+}+\mathrm{HD} \rightarrow \mathrm{H}_{2} \mathrm{D}^{+}+\mathrm{H}_{2}+\Delta E_{1}$.

Neutral molecules like $\mathrm{CO}$ can destroy $\mathrm{H}_{2} \mathrm{D}^{+}$, thereby lowering the deuterium enhancement. Therefore, the depletion of $\mathrm{CO}$, the second most abundant molecule, from the gas phase can lead to an enhancement in the $\left[\mathrm{H}_{2} \mathrm{D}^{+}\right] /\left[\mathrm{H}_{3}^{+}\right]$ratio and thereby the molecular $\mathrm{D} / \mathrm{H}$ ratios.

Roberts \& Millar (2000b) show that at $10 \mathrm{~K}$, accretion of neutrals onto the dust grains, especially $\mathrm{CO}$, leads to the formation of doubly deuterated molecules such as $\mathrm{NHD}_{2}$ and $\mathrm{D}_{2} \mathrm{CO}$. Based on these arguments, we expect to see a correlation

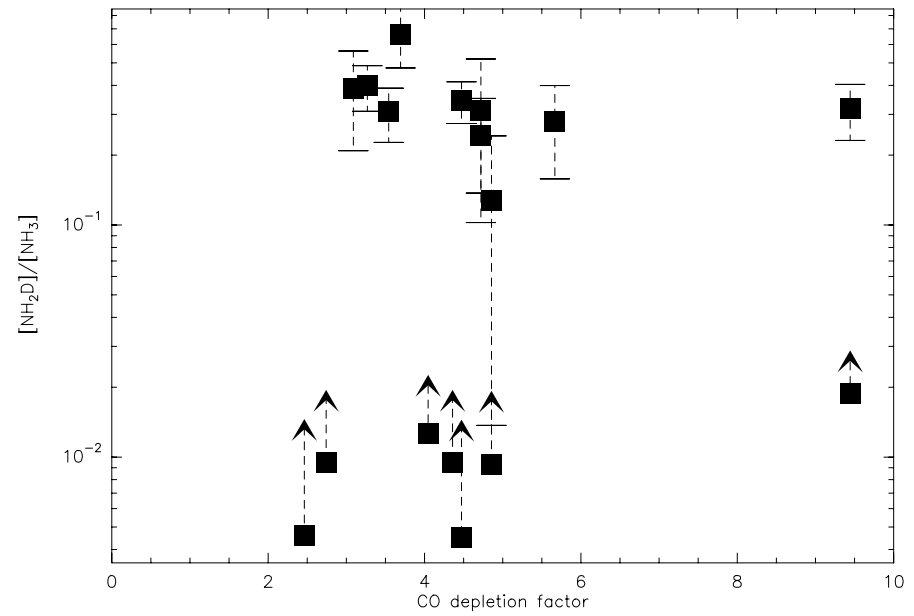

Fig. 3. Comparison of $\mathrm{NH}_{3}$ fractionation and $\mathrm{CO}$ depletion factor for the dense clumps in our sample. The error bar on CO depletion factor is quite large mainly due to the uncertain dust opacities adopted (see Sect. 3.1).

between deuteration and $\mathrm{CO}$ depletion. In Fig. 3, we compare the $\mathrm{NH}_{3}$ fractionation and the degree of $\mathrm{CO}$ depletion (depletion factor). As seen from the correlation plot, there is a large scatter in the values and we do not see any obvious trend of high depletion and deuteration. Nevertheless, the main result is that we find very high deuterium fractionation and $\mathrm{CO}$ depletion. 
Table 6. $\mathrm{H}^{13} \mathrm{CN}$ and $\mathrm{HC}^{15} \mathrm{~N}$ line parameters and column density.

\begin{tabular}{lccccccc}
\hline \hline & \multicolumn{3}{c}{$\mathrm{H}^{13} \mathrm{CN} J=1-0, F=2-1$} & \multicolumn{3}{c}{$\mathrm{HC}^{15} \mathrm{~N} J=1-0$} \\
Source & $\int T_{\mathrm{MB}} \mathrm{d} v^{a}$ & $\Delta v$ & $T_{\mathrm{rot}}$ & $N_{\mathrm{H}^{13} \mathrm{CN}}$ & $\int T_{\mathrm{MB}} \mathrm{d} v$ & $\Delta v$ & $N_{\mathrm{HC}^{15} \mathrm{~N}}$ \\
\hline $\mathrm{K}$ & $\mathrm{K} \mathrm{km} \mathrm{s}^{-1}$ & $\mathrm{~km} \mathrm{~s}^{-1}$ & $\mathrm{~K}$ & $10^{12} \mathrm{~cm}^{-2}$ & $\mathrm{~K} \mathrm{~km} \mathrm{~s}^{-1}$ & $\mathrm{~km} \mathrm{~s}^{-1}$ & $10^{12} \mathrm{~cm}^{-2}$ \\
\hline $\mathrm{G} 10.21-0.31$ & $0.9(0.1)$ & 3.5 & $15.4(1.5)$ & $12.2(1.1)$ & $0.2(<0.1)$ & $1.2(0.4)$ & $0.5(0.1)$ \\
$\mathrm{G} 13.18+0.32$ & $0.7(0.1)$ & 2.4 & $14.5(1.7)$ & $9.3(1.0)$ & $0.6(0.2)$ & $5.6(2.2)$ & $1.3(0.6)$ \\
$\mathrm{G} 15.01-0.67$ & $3.0(0.1)$ & 3.7 & $17.4(1.3)$ & $42.7(2.5)$ & $0.7(0.1)$ & $3.3(0.4)$ & $1.9(0.2)$ \\
$\mathrm{G} 15.01-0.69$ & $7.3(0.2)$ & 3.4 & $24.3(1.5)$ & $129.5(7.1)$ & $2.6(0.2)$ & $2.8(0.3)$ & $8.6(0.9)$ \\
$\mathrm{G} 15.03-0.65$ & $4.8(0.2)$ & 2.7 & $24.3(1.3)$ & $85.2(4.3)$ & $1.6(0.1)$ & $2.4(0.2)$ & $5.3(0.4)$ \\
& $3.6(0.1)$ & 3.6 & $20.4(2.4)$ & $55.9(4.9)$ & $1.3(0.1)$ & $3.6(0.0)$ & $4.4(1.0)$ \\
$\mathrm{G} 23.41-0.23$ & $1.6(0.1)$ & 1.7 & $16.6(2.9)$ & $21.6(2.6)$ & $0.3(0.1)$ & $1.7(0.0)$ & $0.9(0.2)$ \\
$\mathrm{G} 23.42-0.23$ & $1.3(0.1)$ & 2.0 & $18.8(1.8)$ & $18.6(1.4)$ & $0.5(0.1)$ & $2.6(0.5)$ & $1.3(0.2)$ \\
$\mathrm{G} 28.34+0.06 \mathrm{P} 2$ & $1.4(0.1)$ & 3.4 & $19.0(2.0)$ & $20.3(1.7)$ & $0.4(0.1)$ & $2.6(0.4)$ & $1.1(0.2)$ \\
$\mathrm{G} 35.19-1.73$ & $1.9(0.1)$ & 2.4 & $15.7(2.0)$ & $13.8(1.2)$ & $0.7(0.1)$ & $3.7(0.5)$ & $1.8(0.2)$ \\
$\mathrm{G} 79.34+0.33$ & $0.8(<0.1)$ & 1.5 & $17.0(0.9)$ & $27.1(1.3)$ & $0.6(0.1)$ & $2.0(0.3)$ & $1.4(0.2)$ \\
$\mathrm{G} 81.74+0.59$ & $3.2(0.1)$ & 2.8 & $18.4(1.0)$ & $10.0(0.5)$ & $0.2(<0.1)$ & $1.2(0.2)$ & $0.5(0.1)$ \\
\hline
\end{tabular}

${ }^{a}$ Integrated line intensity for the $\mathrm{H}^{13} \mathrm{CN}$ main group alone.

Depletion could well occur on scales much smaller than the beam for sources at the distances typical for these sources (several $\mathrm{kpc}$ ). In that case, the $\mathrm{CO}$ depletion that is measured as an average over the telescope beam is an underestimate of the true depletion. The $\mathrm{NH}_{2} \mathrm{D}$ emission, on the other hand, is probably dominated by the dense clumps. This could explain why the $\mathrm{CO}$ depletion does not appear to track the deuteration in Fig. 3. The uncertainties on the $\mathrm{CO}$ depletion factors are quite large, mainly due to the uncertainty in the $\mathrm{H}_{2}$ column density estimate from the $850 \mu \mathrm{m}$ dust continuum. The largest uncertainty is a factor of 4 in the dust opacity at $850 \mu \mathrm{m}$ (Ossenkopf \& Henning 1994; Krügel \& Siebenmorgen 1994; Draine \& Lee 1984). But we note that with the choice of the Ossenkopf \& Henning opacities, the derived column densities are already lower than for e.g. Savage $\&$ Mathis (1979) dust properties. The two studies other than ours that directly compare $\mathrm{CO}$ depletion and deuterium fraction are by Bacmann et al. (2003) and Crapsi et al. (2005). Bacmann et al. use the $\mathrm{D}_{2} \mathrm{CO}$ to $\mathrm{H}_{2} \mathrm{CO}$ ratios to determine the deuteration. They claim to find a correlation between $\mathrm{D}_{2} \mathrm{CO}$ to $\mathrm{H}_{2} \mathrm{CO}$ ratios and depletion and argue that the presence of a significant amount of $\mathrm{O}$ (which is also an important $\mathrm{H}_{3}^{+}$destroyer) in the gas phase still undepleted might be responsible for the large scatter observed. Crapsi et al. observe $\mathrm{N}_{2} \mathrm{H}^{+}$and $\mathrm{N}_{2} \mathrm{D}^{+}$toward 31 low-mass starless cores and find a good correlation between deuterium fractionation and $\mathrm{CO}$ depletion.

If $f(\mathrm{X})$ is the fractional abundance of species $\mathrm{X}$ relative to $\mathrm{H}_{2}$ and $R(\mathrm{XD})$ is the abundance ratio of $\mathrm{XD}$ relative to $\mathrm{XH}$, then under the assumption of steady-state and equating formation and destruction in Eq. (4), one finds (Millar 2003)

$R\left(\mathrm{H}_{2} \mathrm{D}^{+}\right)=S_{\mathrm{H}_{2} \mathrm{D}^{+}}(T) f(\mathrm{HD})$.

where $S_{\mathrm{H}_{2} \mathrm{D}^{+}}(T)$ is a function of the different rate coefficients. (forward, backward and dissociative recombination rates) given by

$S_{\mathrm{H}_{2} \mathrm{D}^{+}}(T)=\frac{k_{1 f}}{k_{1 r}+\alpha_{1} f(\mathrm{e})+\Sigma k_{\mathrm{M}} f(\mathrm{M})}$.

Here, $k_{1 f}, k_{1 r}$ are the forward and reverse rate coefficients such that $k_{1 r}=k_{1 f} \exp \left(-\Delta E_{1} / \mathrm{T}\right)$ and $\alpha_{1} \propto 1 / \sqrt{T}$ is the dissociative recombination rate, $f(\mathrm{e})$ is the electron fraction and $\Sigma k_{\mathrm{M}}$ is the rate coefficient of $\mathrm{H}_{2} \mathrm{D}^{+}$with species $\mathrm{M} . \Delta E_{1}$ is the exothermal energy ( $220 \mathrm{~K})$, also given in Eq. (4).
At low temperatures $(T<20 \mathrm{~K})$ observed in cold cores, the primary fractionation reaction is dominated by the forward reaction in Eq. (4). At higher temperatures, the reverse reaction becomes important in removing $\mathrm{H}_{2} \mathrm{D}^{+}$very rapidly from the gas phase. Consequently, the decrease in primary fractionation produces a corresponding decrease in secondary fractionation reactions responsible for deuterium enhancements in molecules. Therefore, one naively expects to find an anti-correlation between temperature and deuterium fractionation. In Fig. 4, we plot the $\mathrm{NH}_{3}$ fractionation against the temperature for the high mass candidate pre/protostellar clumps (this paper) and for pre/protostellar clumps in the lower mass regime from the literature. The latest gas-phase predictions for $\left[\mathrm{NH}_{2} \mathrm{D}\right] /\left[\mathrm{NH}_{3}\right]$ (Roueff et al. 2005) are also plotted. This model assumes a density of $n\left(\mathrm{H}_{2}\right)=10^{5} \mathrm{~cm}^{-3}$, carbon and oxygen depletion factors of 5 and 15 respectively while Nitrogen is kept constant. Our data points nicely fill in the missing observed data points between $10-20 \mathrm{~K}$. Clearly the agreement with the model is moderate.

The levels of deuteration do fall off rapidly with increasing temperature above $25 \mathrm{~K}$ (Roueff et al. 2005), but at the low temperatures shown here the dependence is small. The Roueff et al. models employ fixed $\mathrm{H}_{2}$ densities of around $10^{5} \mathrm{~cm}^{-3}$, which would be typical averages over the $40^{\prime \prime}$ beam for our sources. However the $\mathrm{NH}_{2} \mathrm{D}$ emission will be enhanced at the peaks, where higher densities and higher depletion would be expected to produce higher levels of deuteration. Depletion, which increases over time roughly with the increase in density, has a strong influence on deuteration. The final levels of depletion and deuteration depend on the accretion history (e.g. Flower et al. 2005), but it is clear that higher densities or longer timescales result in higher levels of both, e.g. Roberts et al. (2003) derive $\left[\mathrm{NH}_{2} \mathrm{D}\right] /\left[\mathrm{NH}_{3}\right]$ ratios of $0.4-0.8$ for densities of $3 \times 10^{6} \mathrm{~cm}^{-3}$.

However, most of the low $\left[\mathrm{NH}_{2} \mathrm{D}\right] /\left[\mathrm{NH}_{3}\right]$ ratios and interestingly the high temperatures are from the sources where $\mathrm{NH}_{2} \mathrm{D}$ column densities were estimated from the integrated intensity of the line and therefore are really lower limits on the $\left[\mathrm{NH}_{2} \mathrm{D}\right] /\left[\mathrm{NH}_{3}\right]$ ratios. The exact temperature which we measure for a clump of course depends on the combination of hot/cold gas in the beam, which can explain the scatter between $15-18 \mathrm{~K}$ where in some cases we are still seeing a lot of deuteration and in others it has already diminished.

On average, the fractionation observed in sources in our sample is very high. However, note that few sources at temperatures 


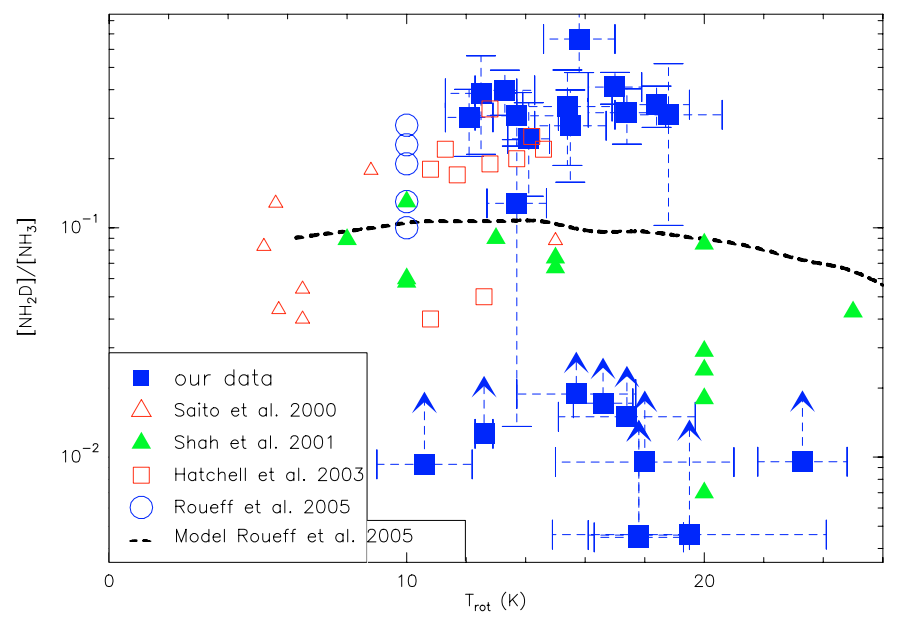

Fig. 4. Plot of $\mathrm{NH}_{3}$ fractionation versus gas temperature as derived from $\mathrm{NH}_{3}$. The dashed line is the latest gas phase model predictions (Roueff et al. 2005). The filled and unfilled squares mark the SCAMPS sources and Hatchell (2003) respectively; filled and unfilled triangles, the values found toward low mass pre/protostellar cores by Shah \& Wootten (2001) and Saito et al. (2000) respectively and the unfilled circles mark Roueff et al. (2005) sources.

above $15 \mathrm{~K}$ show an order of magnitude lower deuteration than the model predictions, while others show extremely high deuterations, much higher than those found in low mass pre/protostellar cores.

There could be three main reasons for the very low fractionation estimated for a few sources. First, one of the main assumption in deriving the $\left[\mathrm{NH}_{3}\right] /\left[\mathrm{NH}_{2} \mathrm{D}\right]$ ratio is that the filling factors for both molecular transitions are the same. Any deviation from this assumption might result in a discrepant ratio. Second, these sources might be relatively more evolved. In such a case, the kinetic temperatures derived from the $\mathrm{NH}_{3}(1,1)$ and $(2,2)$ lines might only be a lower limit. In such cases where the gas temperature is roughly higher than $20 \mathrm{~K}$, the rotational temperature does not represent the real gas temperature. Hence the column density (deuteration) determined may be under estimated. Third, the sources could be chemically young, hence the timescale to reach the high deuteration in these sources is larger than their age. Tafalla \& Santiago (2004) recently discussed such a chemically young low mass core. It is also possible that $\mathrm{NH}_{2} \mathrm{D}$ might be tracing different regions from $\mathrm{NH}_{3}$ and that the temperature derived from $\mathrm{NH}_{3}$ may not be the temperature of $\mathrm{NH}_{2} \mathrm{D}$.

\subsubsection{Upper limits from $\mathrm{NHD}_{2}$}

A simple model for grain surface formation of multiply deuterated molecules is considered by Brown \& Millar (1989a) and predicts the abundances of deuterated species, in order to differentiate between the gas and grain chemistry. According to the Brown and Millar model prediction, the abundances of deuterated species scale as $\left[\mathrm{NHD}_{2}\right] /\left[\mathrm{NH}_{3}\right]=\frac{1}{3}\left(\left[\mathrm{NH}_{2} \mathrm{D}\right] /\left[\mathrm{NH}_{3}\right]\right)^{2}$ for grain surface formation.

Alternatively, Roberts \& Millar (2000a) propose a gas-phase chemistry where the effects of the freeze out of gas phase species onto grains is included and find an enhancement in the fractionation of both singly and doubly deuterated species.

Very deep observations of the $\mathrm{NHD}_{2} 1_{10}-1_{01}$ line for two sources G35.19-1.73 and G81.74+0.59, did not result in any

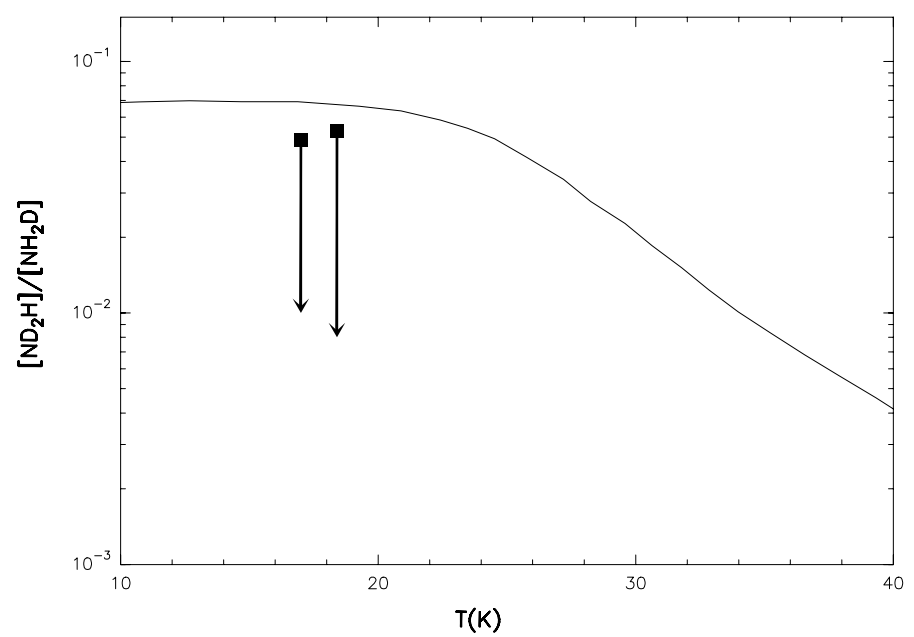

Fig. 5. Comparison of obtained upper limits for G35.19-1.73 and G81.74+0.59 with the gas phase model predictions (Roueff et al. 2005, their model 2).

detection at an rms of $\approx 20 \mathrm{mK}$. Based on the $3 \sigma$ upper limits, we then derive $\left[\mathrm{NHD}_{2}\right] /\left[\mathrm{NH}_{3}\right]$ (see Appendix A) and find that

$\left(\left[\mathrm{NHD}_{2}\right] /\left[\mathrm{NH}_{3}\right]\right)_{\text {observed }}<(0.02)_{\mathrm{G} 35.19} ;(0.02)_{\mathrm{G} 81.74}$

$\left(\frac{1}{3}\left(\left[\mathrm{NH}_{2} \mathrm{D}\right] /\left[\mathrm{NH}_{3}\right]\right)^{2}\right)_{\text {observed }}<(0.06)_{\mathrm{G} 35.19} ;(0.04)_{\mathrm{G} 81.74}$

$\left(\left[\mathrm{NHD}_{2}\right] /\left[\mathrm{NH}_{2} \mathrm{D}\right]\right)_{\mathrm{observed}}<(0.05)_{\mathrm{G} 35.19} ;(0.05)_{\mathrm{G} 81.74}$.

The grain-chemistry model prediction is based purely on probability arguments assuming that the fractionation is proportional to gas-phase atomic $\mathrm{D} / \mathrm{H}$ ratio and that there are three different pathways for inserting the $\mathrm{D}$ atom into the chain of reactions that form multiply deuterated $\mathrm{NH}_{3}$. In spite of the simplicity of the model, the prediction is close to the derived upper limits in both sources.

How do the obtained upper limits compare with the predictions of recent gas-phase deuterium chemistry models? In Fig. 5, we compare the results of model calculations with our observed upper limits for the ratio of $\mathrm{NHD}_{2}$ to $\mathrm{NH}_{2} \mathrm{D}$ (Roueff et al. 2005). We have used their "model 2" which gives the highest molecular fractional abundances for the N-bearing species. In this context it is also interesting to note that while Roueff et al. find that their model underpredicts the $\mathrm{NHD}_{2}$ to $\mathrm{NH}_{2} \mathrm{D}$ ratio in low mass prestellar cores (LDN 134N, LDN 1689N, Barnard 1, LDN 1544), it agrees well with the observed ratio in our sample.

Hence, we cannot reject active grain or gas-phase chemistry on the basis of the observed $\mathrm{NHD}_{2}$ upper limits. While a gas-phase model along with condensation of neutral species onto grain mantles adequately explains moderate deuteration $\left(\mathrm{NH}_{2} \mathrm{D} / \mathrm{NH}_{3}\right)$ observed towards some sources, the very high fractionation we derive remains unexplained. Although we do not understand the grain-surface chemistry, particularly in this case involving deuterated ammonia, a naive extrapolation from the surface-chemistry involving $\mathrm{H}, \mathrm{D}, \mathrm{C}$ and $\mathrm{O}$ and a high grainsurface $\mathrm{D} / \mathrm{H}$ ratio might explain the significant enhancement of deuterated ammonia (Caselli et al. 2004). However, the efficacy of this model is strongly dependent on the gas-phase atomic $\mathrm{D} / \mathrm{H}$ ratio, which in the extreme case of our sample implies an enhancement of $\approx 10^{4}$ over the cosmic $\mathrm{D} / \mathrm{H}$ ratio. 


\subsubsection{Comparison with deuteration in other star forming regions}

Although the exact evolutionary status of the sources (i.e. pre/protostellar) in our sample cannot be determined with certainty, there are several observational evidences that substantiate their high mass pre/protostellar nature. The large line broadening $\left(1<\Delta v<5 \mathrm{~km} \mathrm{~s}^{-1}\right)$ observed in all molecular tracers, together with the high $\mathrm{H}_{2}$ column density $\left(N\left(\mathrm{H}_{2}\right)>10^{23} \mathrm{~cm}^{-2}\right)$ is characteristic of massive star forming regions. These sources have masses between a few hundred to a few thousand solar masses. Their source-averaged $\mathrm{H}_{2}$ densities are a few $10^{5} \mathrm{~cm}^{-3}$ - unlikely for low-mass objects. Moreover, the physical properties of these cores are similar to those associated with UCHII regions (e.g. Thompson et al. 2006). Therefore we expect them to be forming (or on the verge of forming) high-mass stars. The exact nature of these sources will be discussed in a future paper (Hatchell et al. in prep.).

The deuteration of a few tens of percent in ammonia is comparable to the highest which have been measured in the interstellar medium, which occur in low-mass prestellar cores. The related ratio $\left[\mathrm{N}_{2} \mathrm{D}^{+}\right] /\left[\mathrm{N}_{2} \mathrm{H}^{+}\right]$exceeds $20 \%$ in a few prestellar cores including L1544 (Crapsi et al. 2004). High deuterated ammonia abundances have also been measured in early-stage protostars: the $\left[\mathrm{NH}_{2} \mathrm{D}\right] /\left[\mathrm{NH}_{3}\right]$ ratio reaches over $30 \%$ in protostars in Perseus (Hatchell 2003), and detections of triply deuterated ammonia (van der Tak et al. 2002; Lis et al. 2002) and methanol (Parise et al. 2004) have all been in the environments of protostars, though deuteration of $\sim 5 \%$ is more common (Hatchell 2003; Saito et al. 2000; Shah \& Wootten 2001). The high deuteration of our sample supports the idea that these are the high-mass equivalent of prestellar or protostellar cores.

Hot molecular cores, believed to be the early protostellar stage of high mass star formation and potentially a later evolutionary stage of our sources, have a low molecular deuteration fractionation $\sim 10^{-3}$, consistent with ice formation at a higher temperature of 60-80 K and little gas-phase deuteration, as expected at high temperatures (Hatchell et al. 1998, 1999; Roberts et al. 2002). High $\left[\mathrm{NH}_{2} \mathrm{D}\right] /\left[\mathrm{NH}_{3}\right]$ ratios are observed in the Orion region but only in the compact ridge and not the hot core (Turner 1990). If our candidate precluster cores are precursors of hot cores, then the chemistry must radically alter during the later evolution of the hot core, perhaps with continuing accretion at higher temperatures during the early protostellar phase dominating the ice production.

\subsection{Variations in $\mathrm{HCN}$ isotopic abundance}

Let us define the abundance ratio, $R_{\text {iso }}$, as

$R_{\text {iso }}=\frac{{ }^{12} \mathrm{C}}{{ }^{13} \mathrm{C}} \frac{{ }^{15} \mathrm{~N}}{{ }^{14} \mathrm{~N}}$.

$R_{\text {iso }}$ is found to vary with the distance from the Galactic center (Wilson \& Rood 1994; Wielen \& Wilson 1997). The observed trend can be explained by Galactic chemical evolution. Other observational studies (Ikeda et al. 2002; Langer \& Penzias 1993; Dahmen et al. 1995) have found in addition a source-to-source variation of the isotopic ratios among a group of clouds located at nearly the same distance from the galactic centre.

The isotopomeric species of $\mathrm{HCN}, \mathrm{H}^{13} \mathrm{CN}$ and $\mathrm{HC}^{15} \mathrm{~N}$ have been often observed in their $J=1-0$ transition toward various local molecular clouds (Dahmen et al. 1995; Hirota et al. 1998; Ikeda et al. 2002) to estimate the ratio $R_{\text {iso }}$. These lines can be observed with the same receiver and hence the ratio derived will

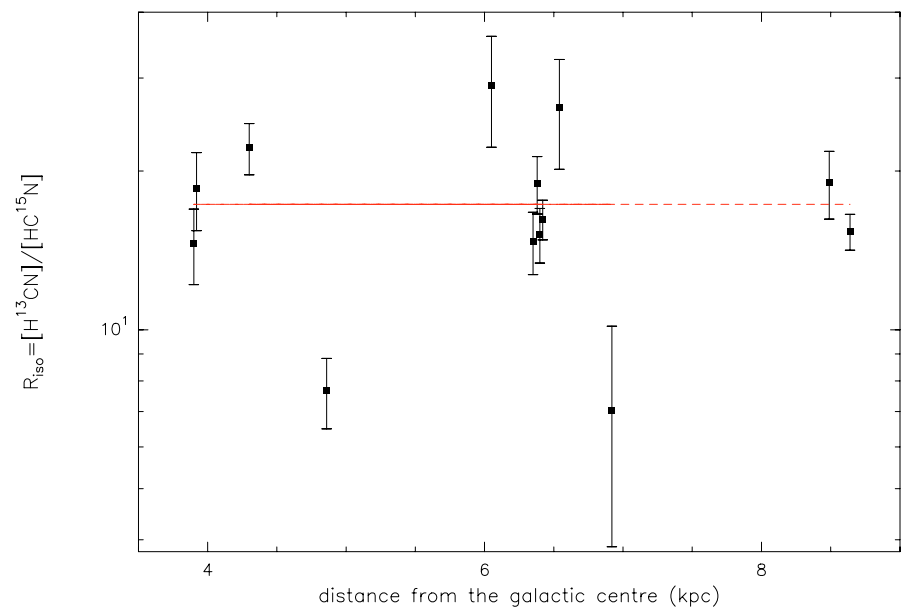

Fig. 6. $\mathrm{H}^{13} \mathrm{CN}$ and $\mathrm{HC}^{15} \mathrm{~N}$ abundance ratio as a function of the distance from the galactic centre. The results are consistent with a single value of $R_{\text {iso }}=17$ shown as the dashed line.

be independent of calibration errors. Moreover, the optical depth can be measured from the three hyperfine components of the $\mathrm{H}^{13} \mathrm{CN}$ line. As mentioned in Sect. $2.5, \mathrm{H}^{13} \mathrm{CN}$ is optically thin in almost all cases and hence the observed ratios must indicate the abundance ratio $R_{\text {iso }}$, since the column density estimates are free from bias due to photon trapping effects.

In Fig. 6 we plot the abundance ratio of $\mathrm{H}^{13} \mathrm{CN}$ and $\mathrm{HC}^{15} \mathrm{~N}$ as a function of distance from the galactic centre for our sources. The ratio of the abundances does not seem to correlate with the distance. However, the formal error bars are too large to be able to make any strong argument against a correlation of $R_{\text {iso }}$ with galactocentric distance.

If we adopt the canonical values for the ${ }^{12} \mathrm{C} /{ }^{13} \mathrm{C}$ of 77 and ${ }^{14} \mathrm{~N} /{ }^{15} \mathrm{~N}$ of 450 toward the local interstellar medium (ISM), then the implied $\mathrm{H}^{13} \mathrm{CN}$ to $\mathrm{HC}^{15} \mathrm{~N}$ abundance ratio is $\sim 6$ (Wilson \& Rood 1994, their Table 4). From our data, we find that most of the values tend to be higher, consistent with a mean value of 17 . This ratio is intermediate between that found toward the galactic centre $(\sim 30)$, an extreme high mass star-forming region and the local ISM $(\sim 6)$ where typically low mass star formation pervades (see again Table 4 of Wilson \& Rood 1994).

The SCAMPS sources are in the close vicinity of massive star forming regions and hence the energetic UV radiation could cause a isotope-selective photo-destruction (Schilke et al. 1992). Chemical fractionation is also likely to play an important role in the observed high values. The high deuterium fractionation we observed must be equally affecting the abundances of $\mathrm{H}^{13} \mathrm{CN}$ and $\mathrm{HC}^{15} \mathrm{~N}$. Therefore, if at all, chemical fractionation were to play a role, it must be attributed to an anomalous fractionation in the isotopes of either $\mathrm{N}$ or $\mathrm{C}$. A high ratio implies either a low ${ }^{15} \mathrm{~N}$ fractionation or ${ }^{13} \mathrm{C}$ fractionation in $\mathrm{HCN}$. As noted earlier in this section, the carbon isotopic ratio is known to vary across the Galaxy (Wilson \& Rood 1994). The ratio is influenced mainly by a) the self-shielding property of $\mathrm{CO}, \mathrm{b})^{13} \mathrm{CO}$ selective isotopic fractionation, and c) stellar winds from stars more massive than the sun, which favours the production of ${ }^{13} \mathrm{C}$ over ${ }^{12} \mathrm{C}$ (Krügel 2003). The deviation from the canonical value in the local ISM could be therefore due to a complex chemistry or environment in these high-mass star forming regions.

Although the number of assumptions involved is considerable, in particular that the two species may not be exactly tracing the same volume of gas as evidenced by the differences in their 
line widths, the ratio we derive clearly indicates the differences in the properties of high mass and low mass star forming regions.

\section{Conclusion}

In this paper, we report the study of physical and chemical properties of a new sample of high-mass pre/protocluster clumps. We have observed 32 sources in the $\mathrm{NH}_{3}(1,1)$ and $(2,2), \mathrm{NH}_{2} \mathrm{D}$ $\left(1_{11}-1_{01}\right), \mathrm{C}^{18} \mathrm{O}(J=1-0$ and $2-1), \mathrm{H}^{13} \mathrm{CN}$ and $\mathrm{HC}^{15} \mathrm{~N}(J=1-$ $0)$ transitions. We have clear detections of $\mathrm{NH}_{2} \mathrm{D}$ in 22 sources and $100 \%$ detection in $\mathrm{NH}_{3}$.

Our observations suggest large deuterium enhancements (up to $66 \%$ ), the largest reported so far. We also derived the amount of molecular depletion by comparing the $\mathrm{C}^{18} \mathrm{O}$ column density with the $\mathrm{H}_{2}$ column density derived from dust continuum observations, and we find that the degree of $\mathrm{CO}$ depletion is a factor 2-12 higher compared to the canonical value for its abundance. The derived abundance ratio of $\mathrm{H}^{13} \mathrm{CN}$ to $\mathrm{HC}^{15} \mathrm{~N}$ is indicative of a very high ${ }^{13} \mathrm{C} /{ }^{12} \mathrm{C}$ ratio, discrepant from that found toward the local ISM. These properties clearly reflect the complex chemistry in regions of high mass star formation.

This study shows that depletion and high deuteration exist towards massive cold cores in massive star forming regions and makes them promising candidates for the early phases of massive star formation. In a subsequent paper, we will discuss the spatial distribution of $\mathrm{NH}_{2} \mathrm{D}$ in two sources observed with high angular resolution, confirming the high deuteration found from this study.

Acknowledgements. T. Pillai was supported for this research through a stipend from the International Max Planck Research School (IMPRS) for Radio and Infrared Astronomy at the University of Bonn. JH was supported at MPIfR Bonn by DFG SFB 494 and holds a PPARC AF at Exeter.

\section{References}

Bachiller, R., Guilloteau, S., \& Kahane, C. 1987, A\&A, 173, 324

Bacmann, A., Lefloch, B., Ceccarelli, C., et al. 2002, A\&A, 389, L6

Bacmann, A., Lefloch, B., Ceccarelli, C., et al. 2003, ApJ, 585, L55

Bergin, E. A., \& Langer, W. D. 1997, ApJ, 486, 316

Beuther, H., \& Shepherd, D. 2005, ArXiv Astrophysics e-prints

Brown, P. D., \& Millar, T. J. 1989a, MNRAS, 240, 25P

Brown, P. D., \& Millar, T. J. 1989b, MNRAS, 237, 661

Carey, S. J., Clark, F. O., Egan, M. P., et al. 1998, ApJ, 508, 721

Caselli, P., Walmsley, C. M., Tafalla, M., Dore, L., \& Myers, P. C. 1999, ApJ, 523, L165

Caselli, P., van der Tak, F. F. S., Ceccarelli, C., \& Bacmann, A. 2003, A\&A, 403, L37

Caselli, P., Stantcheva, T., \& Herbst, E. 2004, in The Dense Interstellar Medium in Galaxies, ed. S. Pfalzner, C. Kramer, C. Staubmeier, \& A. Heithausen, 479 Cesaroni, R., Churchwell, E., Hofner, P., Walmsley, C. M., \& Kurtz, S. 1994, A\&A, 288, 903

Cesaroni, R., Hofner, P., Walmsley, C. M., \& Churchwell, E. 1998, A\&A, 331, 709
Codella, C., Lorenzani, A., Gallego, A. T., Cesaroni, R., \& Moscadelli, L. 2004, A\&A, 417, 615

Crapsi, A., Caselli, P., Walmsley, C. M., et al. 2004, A\&A, 420, 957

Crapsi, A., Caselli, P., Walmsley, C. M., et al. 2005, ApJ, 619, 379

Dahmen, G., Wilson, T. L., \& Matteucci, F. 1995, A\&A, 295, 194

Draine, B. T., \& Lee, H. M. 1984, ApJ, 285, 89

Flower, D. R., Pineau Des Forêts, G., \& Walmsley, C. M. 2005, A\&A, 436, 933

Forveille, T., Guilloteau, S., \& Lucas, R. 1989, Grenoble: IRAM

Frerking, M. A., Langer, W. D., \& Wilson, R. W. 1982, ApJ, 262, 590

Hatchell, J. 2003, A\&A, 403, L25

Hatchell, J., Millar, T. J., \& Rodgers, S. D. 1998, A\&A, 332, 695

Hatchell, J., Roberts, H., \& Millar, T. J. 1999, A\&A, 346, 227

Hirota, T., Yamamoto, S., Mikami, H., \& Ohishi, M. 1998, ApJ, 503, 717

Ho, P. T. P., \& Townes, C. H. 1983, ARA\&A, 21, 239

Ikeda, M., Hirota, T., \& Yamamoto, S. 2002, ApJ, 575, 250

Kramer, C., Alves, J., Lada, C. J., et al. 1999, A\&A, 342, 257

Krügel, E. 2003, The physics of interstellar dust IoP Series in astronomy and astrophysics (Bristol, UK: The Institute of Physics)

Krügel, E., \& Siebenmorgen, R. 1994, A\&A, 288, 929

Langer, W. D., \& Penzias, A. A. 1993, ApJ, 408, 539

Launhardt, R. 1996, Ph.D. Thesis

Lis, D. C., Roueff, E., Gerin, M., et al. 2002, ApJ, 571, L55

Menten, K. M., Pillai, T., \& Wyrowski, F. 2005, in IAU Symp., 23

Millar, T. J. 2002, Planet. Space Sci., 50, 1189

Millar, T. J. 2003, Space Sci. Rev., 106, 73

Müller, H. S. P., Schlöder, F., Stutzki, J., \& Winnewisser, G. 2005, J. Molec. Structure, 742, 215

Öberg, K. I., van Broekhuizen, F., Fraser, H. J., et al. 2005, ApJ, 621, L33

Ossenkopf, V., \& Henning, T. 1994, A\&A, 291, 943

Parise, B., Castets, A., Herbst, E., et al. 2004, A\&A, 416, 159

Pickett, H. M., Poynter, R. L., Cohen, E. A., et al. 1998, J. Quant. Spectrosc. Radiat. Transf., 60, 883

Pillai, T., Wyrowski, F., Carey, S. J., \& Menten, K. M. 2006, A\&A, 450, 569

Roberts, H., \& Millar, T. J. 2000a, A\&A, 364, 780

Roberts, H., \& Millar, T. J. 2000b, A\&A, 361, 388

Roberts, H., Fuller, G. A., Millar, T. J., Hatchell, J., \& Buckle, J. V. 2002, A\&A, 381,1026

Roberts, H., Herbst, E., \& Millar, T. J. 2003, ApJ, 591, L41

Rodgers, S. D., \& Charnley, S. B. 2001, ApJ, 553, 613

Roueff, E., Lis, D. C., van der Tak, F. F. S., Gerin, M., \& Goldsmith, P. F. 2005, A\&A, 438, 585

Saito, S., Ozeki, H., Ohishi, M., \& Yamamoto, S. 2000, ApJ, 535, 227

Savage, B. D., \& Mathis, J. S. 1979, ARA\&A, 17, 73

Savva, D., Little, L. T., Phillips, R. R., \& Gibb, A. G. 2003, MNRAS, 343, 259

Schilke, P., Walmsley, C. M., Pineau Des Forets, G., et al. 1992, A\&A, 256, 595

Shah, R. Y., \& Wootten, A. 2001, ApJ, 554, 933

Tafalla, M., Myers, P. C., Caselli, P., \& Walmsley, C. M. 2004, A\&A, 416, 191

Tafalla, M., Myers, P. C., Caselli, P., Walmsley, C. M., \& Comito, C. 2002, ApJ, 569,815

Tafalla, M., \& Santiago, J. 2004, A\&A, 414, L53

Thompson, M. A., Gibb, A. G., Hatchell, J. H., Wyrowski, F., \& Pillai, T. 2005, in The Dusty and Molecular Universe: A Prelude to Herschel and ALMA, 425

Thompson, M. A., Hatchell, J., Walsh, A. J., MacDonald, G. H., \& Millar, T. J. 2006, A\&A, 453, 1003

Turner, B. E. 1990, ApJ, 362, L29

van der Tak, F. F. S., Schilke, P., Müller, H. S. P., et al. 2002, A\&A, 388, L53

Walmsley, C. M., \& Ungerechts, H. 1983, A\&A, 122, 164

Watson, W. D. 1976, Rev. Mod. Phys., 48, 513

Wielen, R., \& Wilson, T. L. 1997, A\&A, 326, 139

Wilson, T. L., \& Rood, R. 1994, ARA\&A, 32, 191

Wood, D. O. S., \& Churchwell, E. 1989, ApJS, 69, 831

Wu, L. X., \& Yang, J. 2005, Acta Astron. Sinica, 46, 136 
T. Pillai et al.: Deuteration and depletion in high mass clumps.I., Online Material $p 1$

\section{Online Material}




\section{Appendix A: Radiative transfer equations used for column density determination}

We used the following expressions to determine the column density of the different molecules. The column density is given by,

$N_{\mathrm{tot}}=\frac{3 h \varepsilon_{0}}{2 \pi^{2} S \mu_{g}^{2}} J\left(T_{\mathrm{ex}}\right) Q\left(T_{\mathrm{ex}}\right) \tau \Delta v$,

where, $\Delta v$ is the linewidth, $\varepsilon_{0}$ is the dielectric permittivity, $S \mu_{g}^{2}$ is the line strength multiplied by the dipole moment along the molecular $g$-axis, $\tau$ is the optical depth, $h$ is the Planck constant, $T_{\text {ex }}$ is the excitation temperature and $Q\left(T_{\mathrm{ex}}\right)$ is the partition function.

Here, $J\left(T_{\mathrm{ex}}\right)$ is defined as

$J\left(T_{\mathrm{ex}}\right)=\frac{\exp \left(E_{\mathrm{u}} / k T_{\mathrm{ex}}\right)}{\exp \left(h v / k T_{\mathrm{ex}}\right)-1}$,

where $E_{\mathrm{u}}$ is the upper energy level, $k$ is the Boltzmann constant and $v$ is the frequency of observed transition. We assume that the excitation temperature is the same for all rotational levels. Equation (A.1) is valid for the optically thick case.

In the optically thin case,

$N_{\mathrm{tot}}=\frac{3 h \varepsilon_{0}}{2 \pi^{2} S \mu_{g}^{2}} \frac{J\left(T_{\mathrm{ex}}\right) Q\left(T_{\mathrm{ex}}\right) W}{J_{v}\left(T_{\mathrm{ex}}\right)-J_{v}(2.7)}$,

where $\mathrm{W}$ is the integrated intensity and $J_{v}(T)$ is defined as $J_{v}(T)=\frac{h v / k}{\exp \left(h v / k T_{\text {ex }}\right)-1}$.

The $\mathrm{NH}_{2} \mathrm{D}$ partition function is determined by considering the contribution of the different energy levels from $J=0$ to $J=2$, while the metastable levels from $(J, K=1,1)$ to $(J, K=$ 3,3 ) have been considered for $\mathrm{NH}_{3}$.

For other molecular species, the partition function $Q\left(T_{\mathrm{ex}}\right)$ at temperature $T_{\mathrm{ex}}$ is estimated as $Q\left(T_{\mathrm{ex}}\right)=\alpha T^{\beta}$, where $\alpha$ and $\beta$ are the best fit parameters from a fit to the partition function obtained from JPL catalogue at different excitation temperatures from $10-300 \mathrm{~K}$.

In Table A.1, we give the dipole moments we used for different molecules and the partition function, $Q\left(T_{\mathrm{ex}}\right)$ at temperature $T_{\mathrm{ex}}=15 \mathrm{~K}$.

By fitting the main and the hyperfine components of the $(1,1)$ line and the main component of the $(2,2)$ line, we obtain the rotational temperature. The kinetic temperature and $\mathrm{NH}_{3}$ column density, have been derived using the standard formulation for $\mathrm{NH}_{3}$ spectra (Bachiller et al. 1987). The $\mathrm{NH}_{3}$ column density has been estimated assuming that the level populations are thermalised, i.e., $T_{\mathrm{ex}}=T_{\text {rot }}$. A similar assumption holds for our estimation of $\mathrm{NH}_{2} \mathrm{D}$ column densities.

The upper limit on the $\mathrm{NHD}_{2}$ column density is estimated from the integrated intensity. This quantity was measured by integrating over a velocity range determined from the $\mathrm{NH}_{2} \mathrm{D}$ line width around the LSR velocity of the source. The $1 \sigma$ uncertainty on this value is estimated from the rms of the noise in the channels where no line emission is expected, and, is given by $\sqrt{N} v_{\text {res }} \sigma$.
Table A.1. Molecular parameters used to estimate $N_{\text {tot }}$.

\begin{tabular}{lcc}
\hline \hline Transition & $S \mu_{g}^{2 a}$ & $Q(15)^{b}$ \\
& $\mathrm{D}$ & \\
\hline $\mathrm{NH}_{2} \mathrm{D} 1_{11}-1_{01}$ (para) & 11.9 & $47.8^{c}$ \\
$\mathrm{NHD}_{2} 1_{10}-1_{01}$ (ortho) & 0.722 & $50.2^{d}$ \\
$\mathrm{H}^{13} \mathrm{CN} J=1-0, F=2-1$ & 14.84 & $22.8^{e}$ \\
$\mathrm{HC} C^{15} \mathrm{~N}=1-0$ & 8.909 & $7.6^{e}$ \\
$\mathrm{C}^{18} \mathrm{O} J=1-0$ & 0.012 & $6.1^{e}$ \\
$\mathrm{C}^{18} \mathrm{O} J=2-1$ & 0.024 & $6.1^{e}$ \\
\hline
\end{tabular}

${ }^{a} S$ is the line strength and $\mu_{g}$ is the dipole moment along the molecular $g$-axis. ${ }^{b}$ the partition function corresponding to a temperature of $15 \mathrm{~K}$, the typical temperature of the sources in the present sample. ${ }^{c}$ the partition function obtained by the summation over the contribution of the different energy levels from $J=0$ to $J=2 .{ }^{d}$ the partition function based on data from the CDMS catalog (Müller et al. 2005). ${ }^{e}$ the partition function based on data from the JPL catalog (Pickett et al. 1998). 
T. Pillai et al.: Deuteration and depletion in high mass clumps.I., Online Material $p 3$

Table A.2. $\mathrm{NH}_{3}$ line parameters with uncertainties (in brackets) from the hyperfine/Gaussian fits.

\begin{tabular}{lcccc}
\hline \hline & & $\mathrm{NH}_{3}(1,1)$ & & $\mathrm{NH}_{3}(2,2)$ \\
Source & $T_{\mathrm{MB}}{ }^{a}$ & $\Delta v^{b}$ & $\tau_{\mathrm{mg}}{ }^{c}$ & $T_{\mathrm{MB}}$ \\
\hline G8.13+0.25 & $(\mathrm{K})$ & $\mathrm{km} \mathrm{s}^{-1}$ & & $(\mathrm{~K})$ \\
& $2.9(0.4)$ & $1.5(0.1)$ & $1.5(0.3)$ & $1.0(0.2)$ \\
G8.68-0.37 & $5.3(0.5)$ & $1.3(0.1)$ & $1.9(0.3)$ & $1.3(0.2)$ \\
& $5.9(0.4)$ & $3.0(0.1)$ & $5.0(0.4)$ & $2.3(0.3)$ \\
G8.71-0.37 & $3.5(0.4)$ & $1.3(0.0)$ & $4.2(0.3)$ & $3.5(0.2)$ \\
G10.15-0.34 & $1.1(0.1)$ & $4.1(0.3)$ & $1.1(0.5)$ & $0.7(0.2)$ \\
G10.21-0.31 & $2.9(0.4)$ & $1.9(0.0)$ & $3.9(0.3)$ & $1.8(0.1)$ \\
G10.21-0.32 & $3.4(0.5)$ & $1.7(0.0)$ & $3.5(0.2)$ & $1.8(0.3)$ \\
& $4.6(0.4)$ & $1.9(0.0)$ & $4.6(0.2)$ & $2.7(0.1)$ \\
G10.61-0.33 & $2.0(0.2)$ & $1.9(0.1)$ & $1.8(0.3)$ & $1.1(0.1)$ \\
G11.11-0.12P1 & $5.1(1.0)$ & $1.3(0.1)$ & $3.5(0.4)$ & $1.9(0.3)$ \\
G11.11-0.12P3 & $3.0(2.2)$ & $2.5(0.1)$ & $2.4(0.2)$ & $1.0(0.2)$ \\
G11.11-0.12P4 & $4.3(0.9)$ & $1.5(0.1)$ & $3.1(0.6)$ & $0.9(0.4)$ \\
G12.19-0.12 & $0.4(0.0)$ & $3.9(0.2)$ & $1.8(0.3)$ & $0.1(0.0)$ \\
G13.18+0.06 & $5.4(0.5)$ & $2.4(0.0)$ & $3.3(0.1)$ & $3.7(0.1)$ \\
G15.01-0.67 & $5.0(0.4)$ & $2.9(0.1)$ & $0.7(0.1)$ & $3.6(0.1)$ \\
G15.01-0.69 & $3.3(0.2)$ & $2.6(0.1)$ & $0.5(0.1)$ & $2.3(0.1)$ \\
G15.03-0.65 & $2.6(0.3)$ & $2.4(0.2)$ & $1.2(0.3)$ & $1.6(0.2)$ \\
& $2.8(0.3)$ & $2.6(0.1)$ & $1.3(0.3)$ & $1.2(0.4)$ \\
G18.17-0.30 & $2.9(0.3)$ & $1.6(0.0)$ & $3.3(0.2)$ & $1.7(0.1)$ \\
G18.21-0.34 & $3.8(0.4)$ & $1.5(0.0)$ & $2.8(0.2)$ & $2.0(0.2)$ \\
G19.30+0.07P1 & $5.0(0.7)$ & $2.0(0.1)$ & $2.1(0.3)$ & $2.7(0.2)$ \\
G19.30+0.07P2 & $3.2(0.6)$ & $1.7(0.1)$ & $2.9(0.5)$ & $1.4(0.3)$ \\
G23.41-0.23 & $3.0(0.3)$ & $2.0(0.1)$ & $1.2(0.1)$ & $1.6(0.2)$ \\
G23.42-0.23 & $2.2(0.3)$ & $1.9(0.1)$ & $1.6(0.2)$ & $1.3(0.1)$ \\
& $1.3(0.4)$ & $1.5(0.1)$ & $0.6(0.4)$ & $0.4(0.1)$ \\
G23.44-0.18 & $3.6(0.2)$ & $3.6(0.1)$ & $1.7(0.1)$ & $2.8(0.1)$ \\
G27.29+0.15 & $1.7(0.2)$ & $2.5(0.1)$ & $1.6(0.2)$ & $0.9(0.1)$ \\
G27.31+0.18 & $1.0(0.2)$ & $2.1(0.1)$ & $2.1(0.4)$ & $0.6(0.1)$ \\
G28.34+0.06P1 & $2.7(0.5)$ & $2.7(0.0)$ & $2.0(0.1)$ & $1.3(0.1)$ \\
G28.34+0.06P2 & $2.8(0.5)$ & $2.0(0.1)$ & $2.7(0.3)$ & $1.5(0.2)$ \\
G29.97-0.05 & $0.8(0.2)$ & $2.0(0.0)$ & $2.4(0.2)$ & $0.3(0.0)$ \\
G33.71-0.01 & $2.0(0.4)$ & $2.4(0.2)$ & $2.9(0.6)$ & $1.5(0.2)$ \\
G34.81-0.28 & $4.7(0.5)$ & $1.4(0.0)$ & $1.6(0.2)$ & $2.2(0.1)$ \\
G35.19-1.73 & $4.7(0.4)$ & $1.6(0.0)$ & $1.8(0.1)$ & $2.4(0.1)$ \\
G79.34+0.33 & $2.4(0.3)$ & $1.2(0.0)$ & $1.5(0.1)$ & $0.7(0.1)$ \\
G81.74+0.59 & $6.1(0.6)$ & $2.2(0.0)$ & $1.3(0.1)$ & $3.2(0.1)$ \\
\hline & & &
\end{tabular}

${ }^{a}$ Main beam brightness temperature from gaussian fit. ${ }^{b}$ FWHM from the hyperfine fits. ${ }^{c}$ optical depth of the main group of hyperfines from the hyperfine fits.

Note: Five sources clearly have at least 2 velocity components, hence the fit parameters have been determined for each component. Similar notations are used for other molecules elsewhere in this paper. The parameter has been fixed for the fit for those entries with no error indicated. 
T. Pillai et al.: Deuteration and depletion in high mass clumps.I., Online Material p 4

Table A.3. $\mathrm{NH}_{2} \mathrm{D}$ line parameters with uncertainties (in brackets) from the hyperfine/Gaussian fits.

\begin{tabular}{lccc}
\hline \hline & \multicolumn{3}{c}{$\mathrm{NH}_{2} \mathrm{D} 85.93 \mathrm{GHz}$} \\
Source & $\int T_{\mathrm{MB}} \mathrm{d} v^{a}$ & $\Delta v^{b}$ & $\tau_{\text {tot }}{ }^{c}$ \\
\hline G8.13+0.25 & $0.5(<0.1)$ & $1.3(0.3)$ & - \\
& $0.7(<0.1)$ & $1.8(0.9)$ & - \\
G8.68-0.37 & - & $1.2(0.1)$ & $2.2(0.7)$ \\
G8.71-0.37 & - & $1.1(<0.1)$ & $3.7(0.6)$ \\
G10.21-0.31 & - & $1.2(0.1)$ & $3.4(1.4)$ \\
G10.21-0.32 & - & $1.4(0.1)$ & $2.3(1.0)$ \\
G10.61-0.33 & $0.3(<0.1)$ & $1.5(0.2)$ & - \\
G11.11-0.12P1 & - & $1.0(0.1)$ & $3.6(1.5)$ \\
G11.11-0.12P4 & $0.4(0.1)$ & $1.3(0.2)$ & - \\
G12.19-0.12 & $1.5(0.1)$ & $3.6(0.5)$ & - \\
G13.18+0.06 & - & $2.2(0.1)$ & $2.0(0.5)$ \\
G18.17-0.30 & - & $1.5(0.1)$ & $4.4(1.1)$ \\
G18.21-0.34 & - & $1.3(0.1)$ & $2.0(0.8)$ \\
G23.41-0.23 & - & $1.7(0.2)$ & $2.1(1.3)$ \\
G23.44-0.18 & $1.4(0.1)$ & $3.1(0.2)$ & - \\
G27.29+0.15 & $0.4(0.1)$ & $1.5(0.4)$ & - \\
G28.34+0.06P2 & $1.2(0.1)$ & $1.8(0.2)$ & - \\
G29.97-0.05 & - & $1.4(0.1)$ & $3.0(0.6)$ \\
G33.71-0.01 & $0.4(<0.1)$ & $1.6(0.3)$ & - \\
G34.81-0.28 & $0.8(0.1)$ & $1.7(0.1)$ & - \\
G35.19-1.73 & - & $1.4(<0.1)$ & $2.7(0.4)$ \\
G79.34+0.33 & - & $1.0(0.9)$ & $1.1(0.1)$ \\
G81.74+0.59 & - & $2.8(0.1)$ & $1.5(0.3)$ \\
\hline
\end{tabular}

${ }^{a}$ Integrated intensity of the line from the Gaussian fits. ${ }^{b}$ FWHM from the hyperfine fits. ${ }^{c}$ Total optical depth from the hyperfine fits. Note: Similar notations are used for other molecules elsewhere in this paper. The parameter has been fixed for the fit for those entries with no error indicated. 
T. Pillai et al.: Deuteration and depletion in high mass clumps.I., Online Material p 5
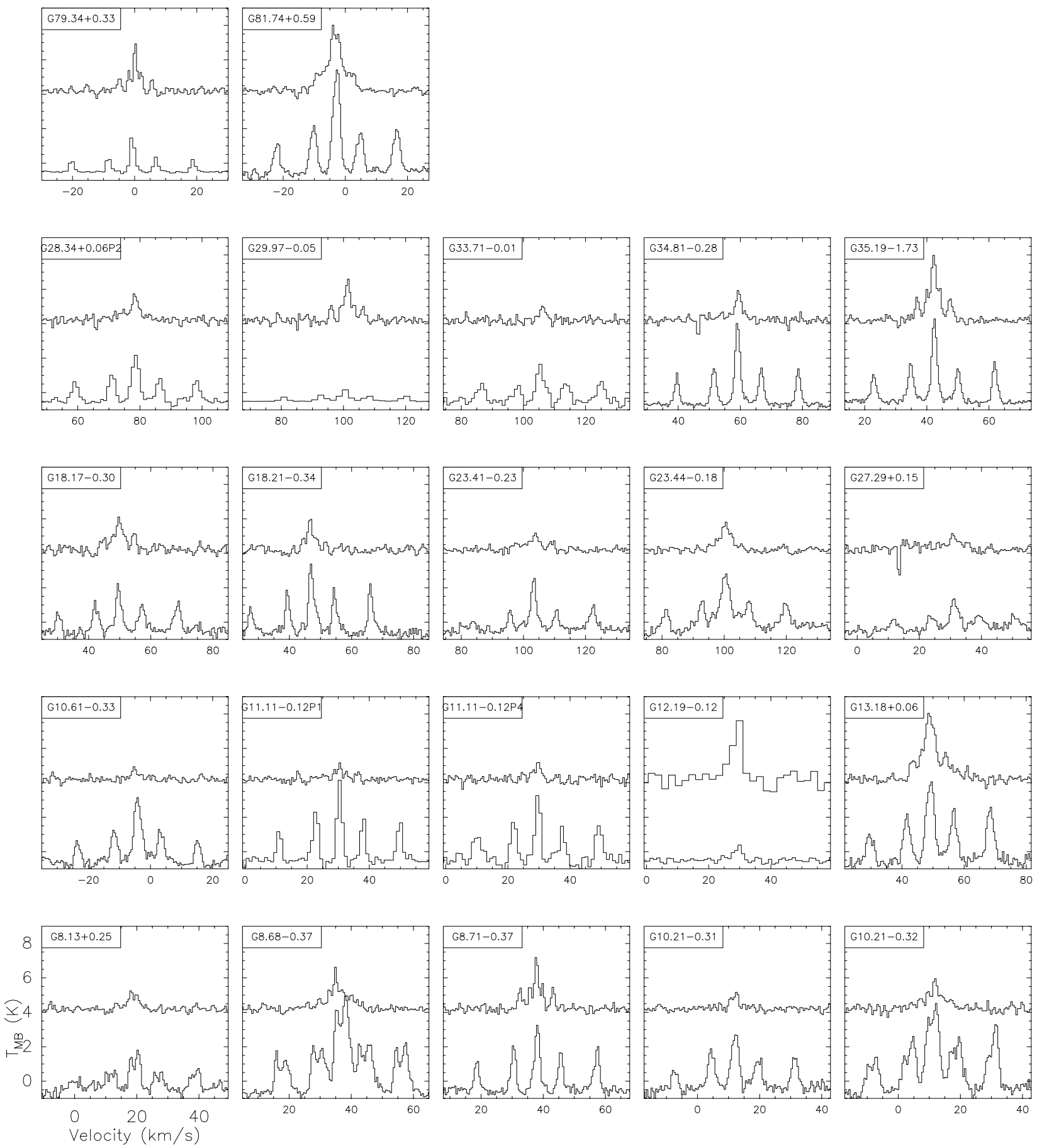

Fig. A.1. Lower spectrum in each panel: Effelsberg $100 \mathrm{~m}$ observation of the $\mathrm{NH}_{3}(1,1)$ emission. Upper spectrum in each panel: $30 \mathrm{~m}$ observation of the $\mathrm{NH}_{2} \mathrm{D}$ at $85.9 \mathrm{GHz}$. The $\mathrm{NH}_{2} \mathrm{D}$ is scaled by a factor 5.0 to amplify the emission relative to $\mathrm{NH}_{3}$ in the absolute (brightness temperature) units. 
T. Pillai et al.: Deuteration and depletion in high mass clumps.I., Online Material p 6
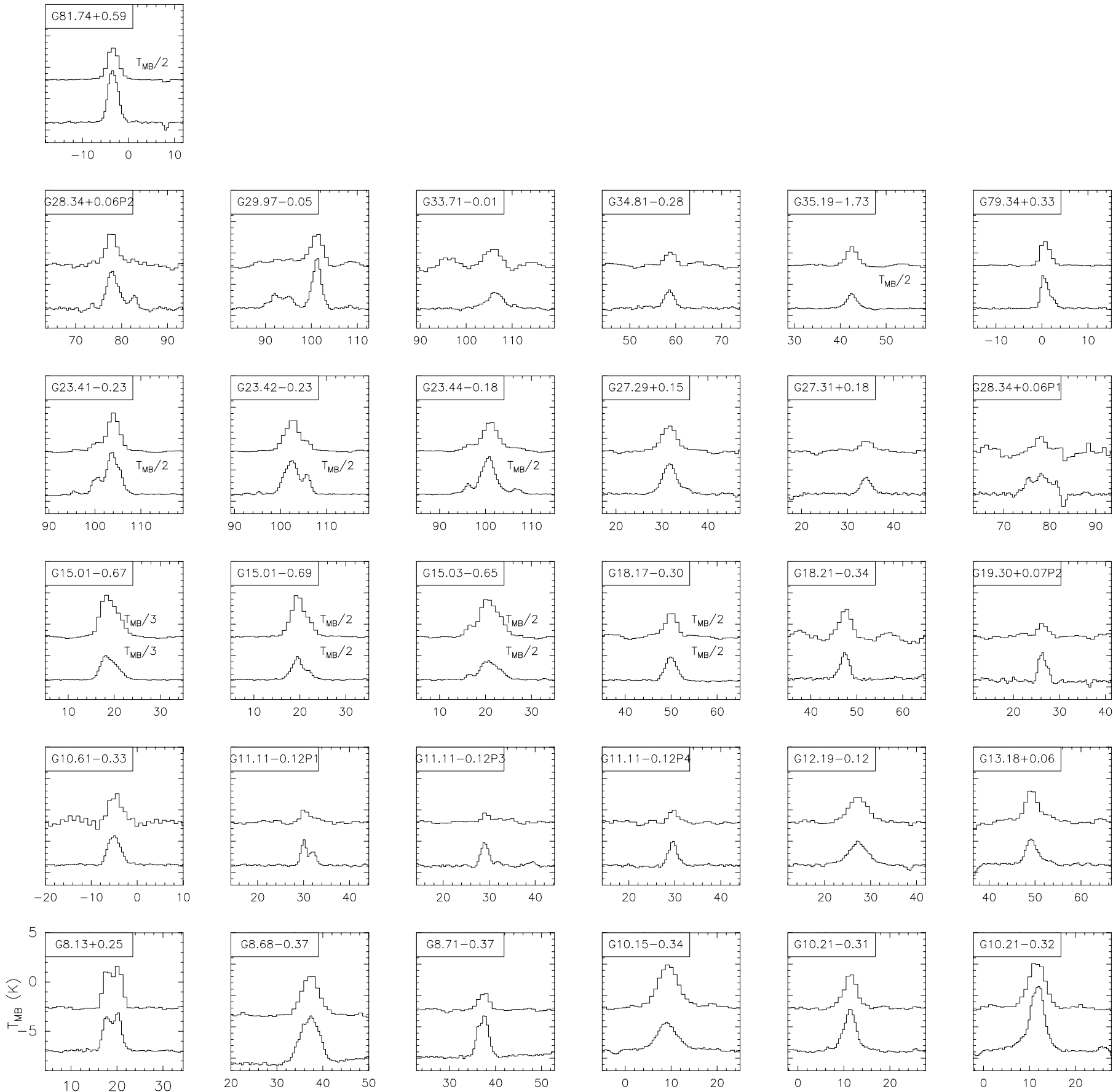

Fig. A.2. Lower spectrum: $30 \mathrm{~m} \mathrm{C}^{18} \mathrm{O}(J=1-0)$. Upper spectrum: $30 \mathrm{~m} \mathrm{C}^{18} \mathrm{O}(J=2-1)$. The velocity range is $\pm 15 \mathrm{~km} \mathrm{~s}^{-1}$ of the systemic velocity. 
T. Pillai et al.: Deuteration and depletion in high mass clumps.I., Online Material $p 7$

Table A.4. $\mathrm{C}^{18} \mathrm{O}(J=1-0)$ and $(J=2-1)$ line parameters.

\begin{tabular}{|c|c|c|}
\hline Source & $\begin{array}{c}\mathrm{C}^{18} \mathrm{O}(J=1-0) \\
\int T_{\mathrm{MB}} \mathrm{d} v\end{array}$ & $\begin{array}{c}\mathrm{C}^{18} \mathrm{O}(J=2-1) \\
\int T_{\mathrm{MB}} \mathrm{d} v\end{array}$ \\
\hline & $\mathrm{K} \mathrm{km} \mathrm{s}^{-1}$ & $\mathrm{~K} \mathrm{~km} \mathrm{~s}^{-1}$ \\
\hline G8.13+0.25 & $15.7(0.1)$ & $27.6(0.5)$ \\
\hline G8.68-0.37 & $24.9(0.9)$ & $31.9(1.7)$ \\
\hline G8.71-0.37 & $13.5(0.4)$ & $5.9(0.7)$ \\
\hline G10.15-0.34 & $15.6(0.3)$ & $37.4(0.5)$ \\
\hline G10.21-0.31 & $14.9(0.2)$ & $19.0(0.4)$ \\
\hline G10.21-0.32 & $27.5(0.3)$ & $33.2(0.6)$ \\
\hline G10.61-0.33 & $10.6(0.3)$ & $14.7(0.5)$ \\
\hline G11.11-0.12P1 & $6.1(0.1)$ & $5.5(0.4)$ \\
\hline G11.11-0.12P3 & $5.1(0.3)$ & $5.1(0.5)$ \\
\hline G11.11-0.12P4 & $5.1(0.2)$ & $4.8(0.5)$ \\
\hline G12.19-0.12 & $12.0(0.2)$ & $20.8(0.4)$ \\
\hline G13.18+0.06 & $9.7(0.5)$ & $20.9(0.8)$ \\
\hline G15.01-0.67 & $31.0(0.2)$ & $91.7(1.0)$ \\
\hline G15.01-0.69 & $16.2(0.2)$ & $53.7(0.5)$ \\
\hline G15.03-0.65 & $19.8(0.2)$ & $65.5(0.6)$ \\
\hline G18.17-0.30 & $13.1(0.7)$ & $26.5(1.3)$ \\
\hline G18.21-0.34 & $7.3(0.4)$ & $12.0(1.3)$ \\
\hline $\mathrm{G} 19.30+0.07 \mathrm{P} 1^{a}$ & $1.1(0.4)$ & - \\
\hline G19.30+0.07P2 & $4.1(0.4)$ & $6.6(0.7)$ \\
\hline G23.41-0.23 & $35.2(0.8)$ & $44.5(1.0)$ \\
\hline G23.42-0.23 & $31.5(0.3)$ & $43.1(0.5)$ \\
\hline G23.44-0.18 & $31.1(0.5)$ & $43.4(0.6)$ \\
\hline $\mathrm{G} 27.29+0.15$ & $12.4(0.2)$ & $16.6(0.5)$ \\
\hline $\mathrm{G} 27.31+0.18$ & $4.9(0.2)$ & $6.1(0.5)$ \\
\hline $\mathrm{G} 28.34+0.06 \mathrm{P} 1$ & $11.3(0.4)$ & $6.3(1.5)$ \\
\hline $\mathrm{G} 28.34+0.06 \mathrm{P} 2$ & $14.4(0.5)$ & $19.1(0.7)$ \\
\hline G29.97-0.05 & $12.9(0.7)$ & $18.2(0.9)$ \\
\hline G33.71-0.01 & $7.4(0.2)$ & $9.0(1.3)$ \\
\hline G34.81-0.28 & $5.4(0.3)$ & $6.6(0.6)$ \\
\hline G35.19-1.73 & $8.4(0.2)$ & $15.8(0.8)$ \\
\hline G79.34+0.33 & $7.8(0.1)$ & $9.9(0.2)$ \\
\hline G81.74+0.59 & $14.9(0.2)$ & $32.4(0.4)$ \\
\hline
\end{tabular}

$\int T_{\mathrm{MB}} \mathrm{d} v$ is the integrated intensity over $\pm 5 \mathrm{~km} \mathrm{~s}^{-1}$ of the brightest $\mathrm{C}^{18} \mathrm{O}(J=1-0)$ peak. ${ }^{a} \mathrm{C}^{18} \mathrm{O}(J=2-1)$ data suffered from baseline ripples. 
T. Pillai et al.: Deuteration and depletion in high mass clumps.I., Online Material $p 8$
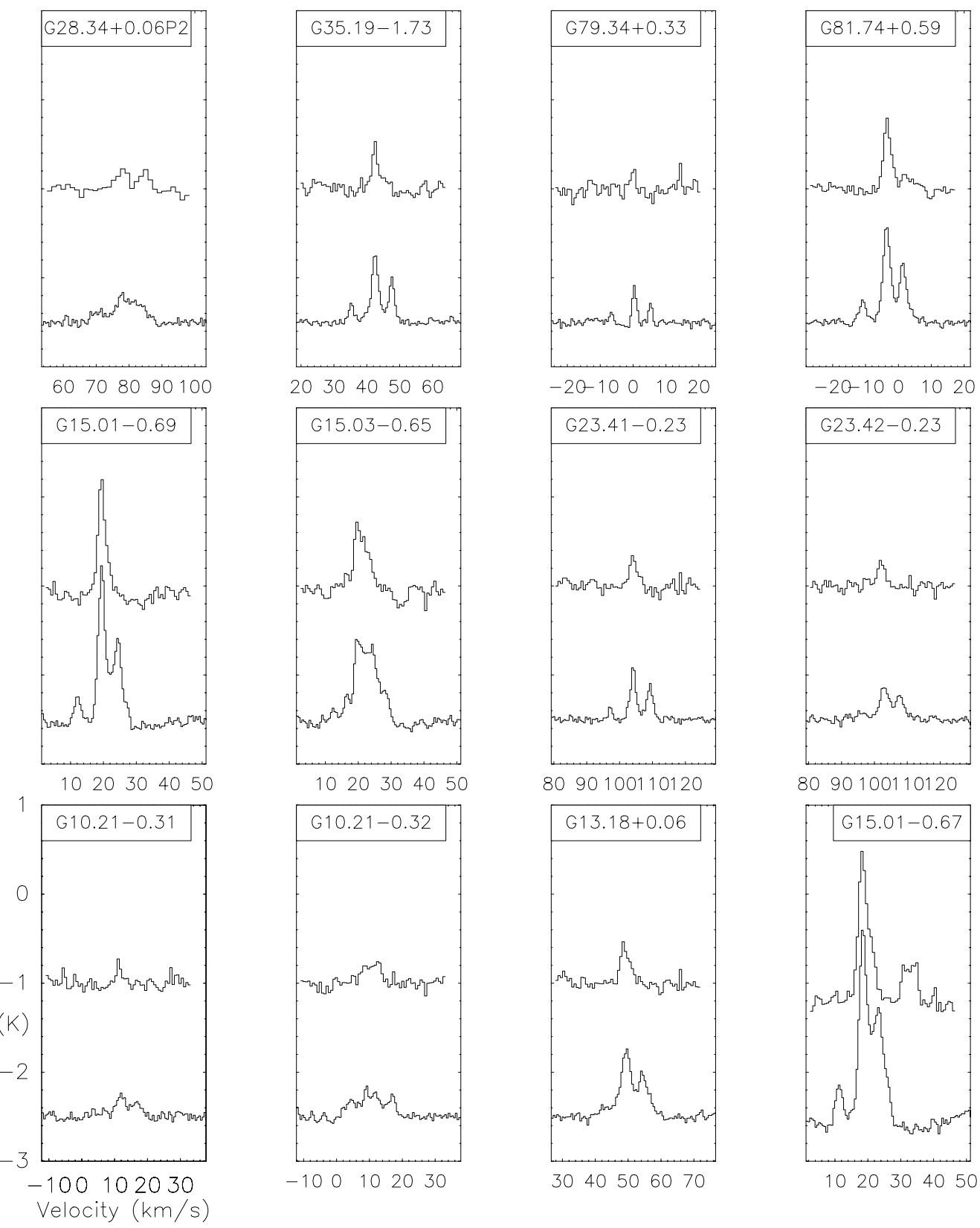

Fig. A.3. Lower spectrum: $30 \mathrm{~m}$ observation of $\mathrm{H}^{13} \mathrm{CN}$. Upper spectrum: $30 \mathrm{~m}$ observation of $\mathrm{HC}^{15} \mathrm{~N}$. The $\mathrm{HC}^{15} \mathrm{~N}$ is scaled by a factor 2 to amplify the emission relative to $\mathrm{H}^{13} \mathrm{CN}$ in the absolute units. The velocity range is $\pm 25 \mathrm{~km} \mathrm{~s}^{-1}$ of the systemic velocity. 